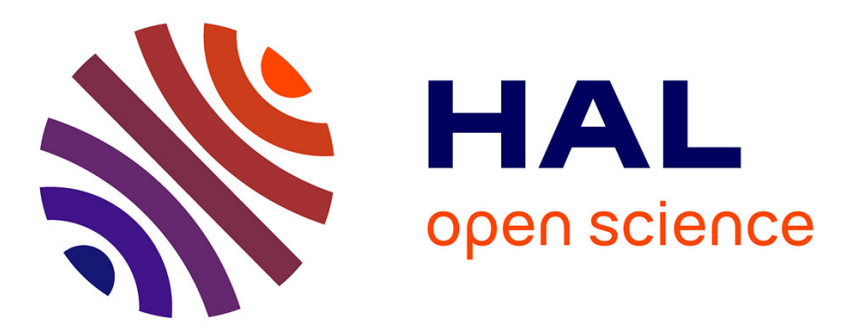

\title{
Quasi-optimal nonconforming approximation of elliptic PDES with contrasted coefficients and $H^{1+r}, r>0$, regularity
}

Alexandre Ern, Jean-Luc Guermond

\section{- To cite this version:}

Alexandre Ern, Jean-Luc Guermond. Quasi-optimal nonconforming approximation of elliptic PDES with contrasted coefficients and $H^{1+r}, r>0$, regularity. 2021. hal-01964299v2

\section{HAL Id: hal-01964299 \\ https://hal.science/hal-01964299v2}

Preprint submitted on 3 May 2021 (v2), last revised 24 Nov 2021 (v3)

HAL is a multi-disciplinary open access archive for the deposit and dissemination of scientific research documents, whether they are published or not. The documents may come from teaching and research institutions in France or abroad, or from public or private research centers.
L'archive ouverte pluridisciplinaire HAL, est destinée au dépôt et à la diffusion de documents scientifiques de niveau recherche, publiés ou non, émanant des établissements d'enseignement et de recherche français ou étrangers, des laboratoires publics ou privés. 


\title{
QUASI-OPTIMAL NONCONFORMING APPROXIMATION OF ELLIPTIC PDES WITH CONTRASTED COEFFICIENTS AND $H^{1+r}$, $r>0$, REGULARITY*
}

\author{
ALEXANDRE ERN ${ }^{\dagger}$ AND JEAN-LUC GUERMOND;
}

\begin{abstract}
In this paper, we investigate the approximation of a diffusion model problem with contrasted diffusivity for various nonconforming approximation methods. The essential difficulty is that the Sobolev smoothness index of the exact solution may be just barely larger than 1 . The lack of smoothness is handled by giving a weak meaning to the normal derivative of the exact solution at the mesh faces. We derive robust and quasi-optimal error estimates. Quasi-optimality means that the approximation error is bounded, up to a generic constant, by the best-approximation error in the discrete trial space, and robustness means that the generic constant is independent of the diffusivity contrast. The error estimates use a mesh-dependent norm that is equivalent, at the discrete level, to the energy norm and that remains bounded as long as the exact solution has a Sobolev index strictly larger than 1. Finally, we briefly show how the analysis can be extended to the Maxwell's equations.
\end{abstract}

Key words. Finite elements, Nonconforming methods, Error estimates, Minimal regularity, Nitsche method, Boundary penalty, Elliptic equations, Maxwell's equations.

AMS subject classifications. 35J25, 65N15, 65N30

This article is dedicated to the memory of Christine Bernardi.

1. Introduction. The objective of the present paper is to revisit and unify the error analysis of various nonconforming approximation techniques applied to a diffusion model problem with contrasted diffusivity. We also briefly show how to extend the analysis to Maxwell's equations.

1.1. Content of the paper. The nonconforming techniques we have in mind are Crouzeix-Raviart finite elements [14], Nitsche's boundary penalty method [32], the interior penalty discontinuous Galerkin (IPDG) method [2], and the hybrid highorder (HHO) methods $[16,18]$ which are closely related to hybridizable discontinuous Galerkin methods [13]. The main difficulty in the error analysis is that owing to the contrast in the diffusivity, the Sobolev smoothness index of the exact solution is barely larger than one. This makes the estimation of the consistency error incurred by nonconforming approximation techniques particularly challenging since the normal derivative of the solution at the mesh faces is not integrable and it is thus not straightforward to give a reasonable meaning to this quantity on each mesh face independently.

The main goal of the present paper is to establish robust and quasi-optimal error estimates by using a mesh-dependent norm that remains bounded as long as the exact solution has a Sobolev smoothness index strictly larger than 1. By quasi-optimality, we mean that the approximation error measured in the augmented norm is bounded, up to a generic constant, by the best-approximation error of the exact solution measured, in the same augmented norm, by members of the discrete trial space. In this

*This material is based upon work supported in part by the National Science Foundation grants DMS-1619892, DMS-1620058, by the Air Force Office of Scientific Research, USAF, under grant/contract number FA9550-18-1-0397, and by the Army Research Office under grant/contract number W911NF-15-1-0517.

${ }^{\dagger}$ Department of Mathematics, Texas A\&M University 3368 TAMU, College Station, TX 77843, USA.

${ }^{\dagger}$ Université Paris-Est, CERMICS (ENPC), 77455 Marne-la-Vallée cedex 2, France and INRIA Paris, 2 rue Simone Iff, 75589 Paris, France. 
paper, we say that an error estimate is robust when the generic constant is independent of the contrast in the diffusivity. This property is important in practice since otherwise, the error estimates become meaningless when the diffusion coefficient is highly contrasted. We emphasize that quasi-optimal error estimates are more informative than the more traditional asymptotic error estimates, which bound the approximation error by terms that optimally decay with the mesh size. Indeed, the former cover the whole computational range whereas the latter only cover the asymptotic range. One key novelty herein is the introduction of a weighted bilinear form that accounts for the default of consistency in all the cases (see (3.12)).

The paper is organized as follows. The model problem under consideration and the discrete setting are introduced in $\S 2$. The weighted bilinear form mentioned above which accounts for the consistency default at the mesh interfaces and boundary faces is defined in $\S 3$. The key results in this section are Lemma 3.3 and Lemma 3.5. We collect in $\S 4$ the error analyses of the approximation of the model problem with the Crouzeix-Raviart approximation, Nitsche's boundary penalty method, the IPDG approximation, and the HHO approximation. To avoid invoking Strang's second lemma, we introduce in $\S 4.1$ a linear form $\delta_{h}$ that measures consistency but does not need the exact solution to be inserted into the arguments of the discrete bilinear form at hand. The weighted bilinear form (3.12) turns out to be an essential tool to deduce robust estimates of the norm of the consistency form $\delta_{h}$ for all the nonconforming methods considered in $\S 4$. Combined with stability, this bound on the consistency error leads to robust and quasi-optimal error estimates formulated using the above mesh-dependent norm. Another salient feature is that the source term is assumed to be only in $L^{q}(D)$, where $q$ is such that $L^{q}(D)$ is continuously embedded in $H^{-1}(D):=$ $\left(H_{0}^{1}(D)\right)^{\prime}$; specifically, this means that $q>2_{*}:=\frac{2 d}{2+d} \geq 1$ (here, $d \geq 2$ is the space dimension).

1.2. Literature overview. Let us put our work in perspective with the literature. Perhaps a bit surprisingly, error estimates for nonconforming approximation methods are rarely presented in a quasi-optimal form in the literature. A key step toward achieving quasi-optimal error estimates has been achieved in Veeser and Zanotti $[34,35]$. Therein, the approximation error and the best-approximation error are both measured using the energy norm and the source term is assumed to be just in the dual space $H^{-1}(D)$. However, at the time of this writing, this setting does not yet cover robust estimates. In the present work, we proceed somewhat differently to obtain robust quasi-optimal error estimates. This is done at the following price: (i) We invoke augmented norms that are stronger than the energy norm, but are, however, compatible with the elliptic regularity theory; (ii) We only consider source terms in the Lebesgue spaces $L^{q}(D)$ with $q>2_{*}:=\frac{2 d}{2+d} \geq 1$, and not in dual Sobolev spaces such as $H^{-1}(D)$; notice though that this regularity is weaker than assuming that source terms are in $L^{2}(D)$, as usually done in the literature, and $L^{q}(D)$ is continuously embedded in $H^{-1}(D):=\left(H_{0}^{1}(D)\right)^{\prime}$ for all $q>2_{*}$.

The traditional approach to tackle the error analysis for nonconforming approximation techniques are Strang's lemmas. However, an important shortcoming of this approach whenever the Sobolev smoothness index of the exact solution is barely larger than one, is that it is not possible to insert the exact solution in the first argument of the discrete bilinear form. To do so, one needs to assume some additional regularity on the exact solution which often goes beyond the regularity provided by the problem at hand. This approach has nevertheless been used by many authors to analyze discontinuous Galerkin (dG) methods (see, e.g., [15, 21] and the references therein). 
One way to overcome the limitations of Strang's Second Lemma has been proposed by Gudi [29]. The key idea consists of introducing a mapping that transforms the discrete test functions into elements of the exact test space. An important property of this operator is that its kernel is composed of discrete (test) functions that are only needed to "stabilize" the discrete bilinear form, but do not contribute to the interpolation properties of the approximation setting. We refer to this mapping as trimming operator. The notion of trimming operator has been used in $\mathrm{Li}$ and Mao [31] to perform the analysis of the Crouzeix-Raviart approximation of the diffusion problem and source term in $L^{2}(D)$ (see e.g., the definitions (5)-(7) and the identity (11) therein). The trimmed error estimate (which is sometimes referred to as "medius analysis" in the literature) has been applied in Gudi [29] to the IPDG approximation of the Laplace equation with a source term in $L^{2}(D)$ and to a fourth-order problem; it has been applied to the Stokes equations in Badia et al. [3] and to the linear elasticity equations in Carstensen and Schedensack [12]. One problem with methods using the trimming operator, though, is that they require constructing $H^{1}$-conforming discrete quasi-approximation operators that do not account for the diffusivity contrast. In general, this entails error estimates with constants that depend on the diffusivity contrast, i.e., these error estimates are not robust. One specific situation where robustness can be achieved though is under the ad hoc assumption of monotonicity around vertices for the diffusivity in the two-dimensional setting; see Bernardi and Verfürth [6, Hyp. 2.7].

It is shown in [25] in the case of Nitsche's boundary penalty method that the dependency of the constants with respect to the diffusivity contrast can be eliminated by introducing an alternative technique based on mollification and an extension of the notion of the normal derivative. The objective of the present paper is to revisit and extend [25]. The analysis presented here is significantly simplified and modified to include the Crouzeix-Raviart approximation, the IPDG approximation, and the HHO approximation. One key novelty is the introduction of the weighted bilinear form (3.12) that accounts for the consistency default in all the cases. The present analysis hinges on two key ideas which are now part of the numerical analysis folklore. To the best of our knowledge, these ideas have been introduced/used in Lemma 4.7 in Amrouche et al. [1], Lemma 2.3 and Corollary 3.1 in Bernardi and Hecht [5] and Lemma 8.2 in Buffa and Perugia [9]. However, as we believe that detailed and convincing proofs of these results are missing in the literature, another purpose of this paper is to fill this gap.

The first key idea is a face-to-cell lifting operator. Such an operator is mentioned in Lemma 4.7 in [1], and its construction is briefly discussed. The weights used in the norms therein, though, cannot give estimates that are uniform with respect to the mesh size. This operator is also mentioned in Lemma 2.3 in [5]. The authors claim that the face-to-cell operator has been constructed in Bernardi and Girault [4, Eq. (5.1)], which is unclear to us. A similar operator is invoked in Lemma 8.2 in [9]. The operator therein is constructed on the reference element $\widehat{K}$ and its stability properties are proved in the Sobolev scale $\left(H^{s}(\widehat{K})\right)_{s \in(0,1)}$. The authors invoke also the Sobolev scale $\left(H^{s}(K)\right)_{s \in(0,1)}$ for arbitrary cells $K$ in a mesh $\mathcal{T}_{h}$ belonging the shape-regular sequence $\left(\mathcal{T}_{h}\right)_{h \in \mathcal{H}}$. The norm equipping $H^{s}(K)$ is not explicitly defined therein, which leads to one statement that looks questionable (see e.g., Eq. (8.11) therein; a fix has been proposed in [8, Lem. A.3]). In particular, it is unclear how to keep track of constants that depend on $K$ when one uses the real interpolation method to define $H^{s}(K)$. In order to unambiguously clarify the status of this face- 
to-cell operator, which is essential for our analysis, and without claiming originality, we give (recall) all the details of its construction in the proof of Lemma 3.1. As in [1, Lem. 4.7], we use the Sobolev-Slobodeckij norm to equip the fractional-order Sobolev spaces; this allows us to track all the constants easily.

The second key idea introduced in the papers referred to above is that of extending the notion of face integrals by using a duality argument together with the face-to-cell operator. The argument is deployed in Corollary 3.3 in [5], but the sketch of the proof has typos (e.g., an average has to be removed to make the inverse estimate in step (1) correct). This corollary is quoted and invoked in Cai et al. [11, Lem. 2.1]; it is the cornerstone of the argumentation therein. This argument is also deployed in Lemma 8.2 in [9]. A similar argument is invoked in [1, Lem. 4.7] in a slightly different context. In all the cases one must use a density argument to complete the proofs, but this argument is omitted and implicitly assumed to hold true in all the above references. We fill this gap in Lemma 3.3 and provide the full argumentation in the proof, including the passage to the limit by density. The proof invokes mollifiers that commute with differential operators and behave properly at the boundary of the domain; these tools have been recently revisited in [22] elaborating on seminal ideas from Schöberl [33].

2. Preliminaries. In this section, we introduce the model problem and the discrete setting for the approximation.

2.1. Model problem. Let $D$ be a Lipschitz domain in $\mathbb{R}^{d}$, which we assume for simplicity to be a polyhedron. We consider the following scalar model problem:

$$
-\nabla \cdot(\lambda \nabla u)=f \quad \text { in } D, \quad \gamma^{\mathrm{g}}(u)=g \quad \text { on } \partial D,
$$

where $\gamma^{\mathrm{g}}: H^{1}(D) \rightarrow H^{\frac{1}{2}}(\partial D)$ is the usual trace map (the superscript ${ }^{\mathrm{g}}$ refers to the gradient), and $g \in H^{\frac{1}{2}}(\partial D)$ is the Dirichlet boundary data. The scalar-valued diffusion coefficient $\lambda \in L^{\infty}(D)$ is assumed to be uniformly bounded from below away from zero. For simplicity, we also assume that $\lambda$ is piecewise constant in $D$, i.e., there is a partition of $D$ into $M$ disjoint Lipschitz polyhedra $D_{1}, \cdots, D_{M}$ s.t. $\lambda_{\mid D_{i}}$ is a positive real number for all $i \in\{1: M\}$. To formalize this structure, we set $\Pi:=\left\{D_{1}, \cdots, D_{M}\right\}$ and $\Lambda(\Pi):=\left\{\lambda \in L^{\infty}(D) \mid \lambda_{\mid D_{i}}>0, \lambda_{\mid D_{i}}\right.$ is constant, $\left.\forall i \in\{1: M\}\right\}$.

It is standard in the literature to assume that $f \in L^{2}(D)$. We are going to relax this hypothesis in this paper by only assuming that $f \in L^{q}(D)$ with $q>\frac{2 d}{2+d}$. Note that $q>1$ since $d \geq 2$. Note also that $L^{q}(D) \hookrightarrow H^{-1}(D)$ since $H_{0}^{1}(D) \hookrightarrow L^{q^{\prime}}(D)$ with the convention that $\frac{1}{q}+\frac{1}{q^{\prime}}=1$. Since $\frac{2 d}{2+d}<2$, we are going to assume without loss of generality that $q \leq 2$.

In the case of the homogeneous Dirichlet condition $(g=0)$, the weak formulation of the model problem (2.1) is as follows:

$$
\left\{\begin{array}{l}
\text { Find } u \in V:=H_{0}^{1}(D) \text { such that } \\
a(u, w)=\ell(w), \quad \forall w \in V,
\end{array}\right.
$$

with the bilinear and linear forms

$$
a(v, w):=\int_{D} \lambda \nabla v \cdot \nabla w \mathrm{~d} x, \quad \ell(w):=\int_{D} f w \mathrm{~d} x .
$$

The bilinear form $a$ is coercive in $V$ owing to the Poincaré-Steklov inequality, and it is also bounded on $V \times V$ owing to the Cauchy-Schwarz inequality. The linear form $\ell$ 
is bounded on $V$ since the Sobolev embedding theorem and Hölder's inequality imply that $|\ell(w)| \leq\|f\|_{L^{q}(D)}\|w\|_{L^{q^{\prime}(D)}} \leq c\|f\|_{L^{q}(D)}\|w\|_{H^{1}(D)}$. Note that $q>\frac{2 d}{2+d}$ is the minimal integrability requirement on $f$ for this boundedness property to hold true. The above coercivity and boundedness properties combined with the Lax-Milgram Lemma imply that (2.2) is well-posed. For the non-homogeneous Dirichlet boundary condition, one invokes the surjectivity of the trace map $\gamma^{\mathrm{g}}$ to infer the existence of a lifting of $g$, say $u_{g} \in H^{1}(D)$ s.t. $\gamma^{\mathrm{g}}\left(u_{g}\right)=g$, and one decomposes the exact solution as $u=u_{g}+u_{0}$ where $u_{0} \in H_{0}^{1}(D)$ solves the weak problem (2.2) with $\ell(w)$ replaced by $\ell_{g}(w)=\ell(w)-a\left(u_{g}, w\right)$. The weak formulation thus modified is well-posed since $\ell_{g}$ is bounded on $H_{0}^{1}(D)$.

The notion of diffusive flux, which is defined as follows, will play an important role in the paper:

$$
\boldsymbol{\sigma}(v):=-\lambda \nabla v \in \boldsymbol{L}^{2}(D), \quad \forall v \in H^{1}(D) .
$$

We use boldface notation to denote vector-valued functions and vectors in $\mathbb{R}^{d}$.

Assumption 2.1 (Elliptic regularity). We assume in the entire paper that for all $q \in\left(\frac{2 d}{2+d}, 2\right]$, there is $r>0$ so that for all $f \in L^{q}(D)$ and all $\lambda \in \Lambda(\Pi)$, the unique solution to (2.1) satisfies $u \in H^{1+r}(D)$.

Assumption 2.1 is reasonable owing to the elliptic regularity theory (see Theorem 3 in Jochmann [30], Lemma 3.2 in Bonito et al. [7] or Bernardi and Verfürth [6]). In general, $r \in\left(0, \frac{1}{2}\right]$ when $u$ is supported on at least two contiguous subdomains where $\lambda$ takes different values; otherwise the normal derivative of $u$ would be continuous across the interface separating the two subdomains in question, and owing to the discontinuity of $\lambda$, the normal component of the diffusive flux $\boldsymbol{\sigma}(u)$ would be discontinuous across the interface, which would contradict the fact that $\boldsymbol{\sigma}(u)$ has a weak divergence. It is, however, possible that $r>\frac{1}{2}$ when the exact solution is supported on one subdomain only. If $r \geq 1$, we notice that one necessarily has $f \in L^{2}(D)$ (since $f_{\mid D_{i}}=\lambda_{\mid D_{i}}(\Delta u)_{D_{i}}$ for all $\left.i \in\{1: M\}\right)$, i.e., it is legitimate to assume that $q=2$ if $r \geq 1$. The paper focuses on the case $r \in\left(0, \frac{1}{2}\right]$.

Lemma 2.2 (Exact solution). Assume that Assumption 2.1 holds true. Then, there exists $p>2$ so that, for all $f \in L^{q}(D)$ and all $\lambda \in \Lambda(\Pi)$, the unique solution to (2.2) satisfies

$$
u \in V_{\mathrm{S}}:=\left\{v \in H_{0}^{1}(D) \mid \boldsymbol{\sigma}(v) \in \boldsymbol{L}^{p}(D), \nabla \cdot \boldsymbol{\sigma}(v) \in L^{q}(D)\right\} .
$$

Proof. The Sobolev embedding theorem implies that there is $p>2$ s.t. $\boldsymbol{H}^{r}(D) \hookrightarrow$ $\boldsymbol{L}^{p}(D)$. Indeed, if $2 r<d$, we have $\boldsymbol{H}^{r}(D) \hookrightarrow \boldsymbol{L}^{s}(D)$ for all $s \in\left[2, \frac{2 d}{d-2 r}\right]$ and we can take $p=\frac{2 d}{d-2 r}>2$, whereas if $2 r \geq d$, we have $\boldsymbol{H}^{r}(D) \hookrightarrow \boldsymbol{H}^{\frac{d}{2}}(D) \hookrightarrow \boldsymbol{L}^{s}(D)$ for all $s \in[2, \infty)$, and we can take any $p>2$. Since $u \in H^{1+r}(D)$, the above argument implies that $\nabla u \in \boldsymbol{L}^{p}(D)$, and since $\lambda$ is piecewise constant and $\boldsymbol{\sigma}(u)=-\lambda \nabla u$, we have $\boldsymbol{\sigma}(u) \in \boldsymbol{L}^{p}(D)$. Moreover, since $\nabla \cdot \boldsymbol{\sigma}(u)=f$ and $f \in L^{q}(D)$, we have $\nabla \cdot \boldsymbol{\sigma}(u) \in L^{q}(D)$.

Remark 2.3 (Extensions). One could also consider lower-order terms in (2.1), e.g., $-\nabla \cdot(\lambda \nabla u)+\boldsymbol{\beta} \cdot \nabla u+\mu u=f$ with $\boldsymbol{\beta} \in \boldsymbol{W}^{1, \infty}(D)$ and $\mu \in L^{\infty}(D)$ s.t. $\mu-\frac{1}{2} \nabla \cdot \boldsymbol{\beta} \geq 0$ a.e. in $D$ (for simplicity). The error analysis presented in this paper still applies provided the lower-order terms are not too large, e.g., $\lambda \geq \max \left(h\|\boldsymbol{\beta}\|_{L^{\infty}(\Omega)}, h^{2}\|\mu\|_{L^{\infty}(D)}\right)$, where $h$ denotes the mesh-size. Standard stabilization techniques have to be invoked 
if the lower-order terms are large when compared to the second-order diffusion operator. Furthermore, the error analysis can be extended to account for a piecewise constant tensor-valued diffusivity $\mathbb{d}$; then, the various constants in the error estimate depend on the square-root of the anisotropy ratios measuring the contrast between the largest and the smallest eigenvalue of $\mathbb{d}$ in each subdomain $D_{i}$. Finally, one can consider that the diffusion tensor $\mathbb{d}$ is piecewise smooth instead of being piecewise constant; a reasonable requirement is that $\mathbb{d}_{\mid D_{i}}$ is Lipschitz for all $i \in\{1: M\}$. This last extension is, however, less straightforward because the discrete diffusive flux is no longer a piecewise polynomial function.

2.2. Discrete setting. We introduce in this section the discrete setting that we are going to use to approximate the solution to $(2.2)$. Let $\mathcal{T}_{h}$ be a mesh from a shaperegular sequence $\left(\mathcal{T}_{h}\right)_{h \in \mathcal{H}}$. Here, $\mathcal{H}$ is a countable set with 0 as unique accumulation point. A generic mesh cell is denoted $K \in \mathcal{T}_{h}$ and is conventionally assumed to be an open set. We also assume that $\mathcal{T}_{h}$ covers each of the subdomains $\left\{D_{i}\right\}_{i \in\{1: M\}}$ exactly so that $\lambda_{K}:=\lambda_{\mid K}$ is constant for all $K \in \mathcal{T}_{h}$. Let $(\widehat{K}, \widehat{P}, \widehat{\Sigma})$ be the reference finite element; we assume that $\mathbb{P}_{k, d} \subset \widehat{P} \subset W^{k+1, \infty}(\widehat{K})$ for some $k \geq 1$. Here, $\mathbb{P}_{k, d}$ is the (real) vector space composed of the $d$-variate polynomials of degree at most $k$. For all $K \in \mathcal{T}_{h}$, let $\boldsymbol{T}_{K}: \widehat{K} \rightarrow K$ be the geometric mapping and let $\psi_{K}^{\mathrm{g}}(v)=v \circ \boldsymbol{T}_{K}$ be the pullback by the geometric mapping. We introduce the broken finite element space

$$
P_{k}^{\mathrm{b}}\left(\mathcal{T}_{h}\right):=\left\{v_{h} \in L^{\infty}(D) \mid v_{h \mid K} \in P_{K}, \forall K \in \mathcal{T}_{h}\right\},
$$

where $P_{K}:=\left(\psi_{K}^{\mathrm{g}}\right)^{-1}(\widehat{P}) \subset W^{k+1, \infty}(K)$. For any function $v_{h} \in P_{k}^{\mathrm{b}}\left(\mathcal{T}_{h}\right)$, we define the broken diffusive flux $\boldsymbol{\sigma}\left(v_{h}\right) \in \boldsymbol{L}^{2}(D)$ by setting $\boldsymbol{\sigma}\left(v_{h}\right)_{\mid K}:=-\lambda_{K} \nabla\left(v_{h \mid K}\right)$ for all $K \in \mathcal{T}_{h}$. Let $W^{1, p}\left(\mathcal{T}_{h}\right):=\left\{v \in L^{p}(D) \mid \nabla\left(v_{\mid K}\right) \in L^{p}(K), \forall K \in \mathcal{T}_{h}\right\}$ and let $\nabla_{h}:$ $W^{1, p}\left(\mathcal{T}_{h}\right) \rightarrow \boldsymbol{L}^{2}(D)$ be the broken gradient operator defined by setting $\left(\nabla_{h} v\right)_{\mid K}:=$ $\nabla\left(v_{\mid K}\right)$ for all $K \in \mathcal{T}_{h}$ and all $v \in W^{1, p}\left(\mathcal{T}_{h}\right)$. Then, we have $\boldsymbol{\sigma}\left(v_{h}\right)=-\lambda \nabla_{h} v_{h}$.

For any cell $K \in \mathcal{T}_{h}, \boldsymbol{n}_{K}$ denotes the unit normal vector on $\partial K$ pointing outward. The symbol $\mathcal{F}_{h}^{\circ}$ denotes the collection of the mesh interfaces and $\mathcal{F}_{h}^{\partial}$ denotes the collection of the mesh faces at the boundary of $D$. We assume that $\mathcal{T}_{h}$ is oriented in a generation-compatible way, and for each mesh face $F \in \mathcal{F}_{h}^{\circ} \cup \mathcal{F}_{h}^{\partial}, \boldsymbol{n}_{F}$ denotes the unit vector orienting $F$. For all $F \in \mathcal{F}_{h}^{\circ}$, the symbols $K_{l}, K_{r} \in \mathcal{T}_{h}$ denote the two cells s.t. $F=\partial K_{l} \cap \partial K_{r}$ and the unit normal vector $\boldsymbol{n}_{F}$ orienting $F$ points from $K_{l}$ to $K_{r}$, i.e., $\boldsymbol{n}_{F}=\boldsymbol{n}_{K_{l} \mid F}=-\boldsymbol{n}_{K_{r} \mid F}$. For all $F \in \mathcal{F}_{h}$, let $\mathcal{T}_{F}$ be the collection of the one or two mesh cells sharing $F$. For all $K \in \mathcal{T}_{h}$, let $\mathcal{F}_{K}$ be the collection of the faces of $K$. For all $K \in \mathcal{T}_{h}$ and all $F \in \mathcal{F}_{K}$, let $\epsilon_{K, F}:=\boldsymbol{n}_{F} \cdot \boldsymbol{n}_{K \mid F}= \pm 1$. The jump across $F \in \mathcal{F}_{h}^{\circ}$ of any function $v \in W^{1,1}\left(\mathcal{T}_{h}\right)$ is defined by setting $\llbracket v \rrbracket_{F}(\boldsymbol{x}):=v_{\mid K_{l}}(\boldsymbol{x})-v_{\mid K_{r}}(\boldsymbol{x})$ for a.e. $\boldsymbol{x} \in F$. If $F \in \mathcal{F}_{h}^{\partial}$, this jump is conventionally defined as the trace on $F$, i.e., $\llbracket v \rrbracket_{F}(\boldsymbol{x}):=v_{\mid K_{l}}(\boldsymbol{x})$ where $F=\partial K_{l} \cap \partial D$. We omit the subscript ${ }_{F}$ in the jump whenever the context is unambiguous.

3. The bilinear form $n_{\sharp}$. In this section, we give a proper meaning to the normal trace of the diffusive flux of the solution to (2.2) over each mesh face. The material presented in $\S 3.1$ and $\S 3.2$ has been introduced in $[25, \S 5.3]$ and is inspired from Amrouche et al. [1, Lem. 4.7], Bernardi and Hecht [5, Cor 3.3], and Buffa and Perugia [9, Lem. 8.2]; it is included here for the sake of completeness. The reader familiar with these techniques is invited to jump to $\S 3.3$ where the weighted bilinear form $n_{\sharp}$ is introduced. This bilinear form is the main tool for the error analysis presented in $\S 4$. 
3.1. Face-to-cell lifting operator. Let us first motivate our approach informally. Let $K \in \mathcal{T}_{h}$ be a mesh cell, let $\mathcal{F}_{K}$ be the collection of all the faces of $K$, and let $F \in \mathcal{F}_{K}$ be a face of $K$. Let $\boldsymbol{v}$ be a vector field defined on $K$. We are looking for (mild) regularity requirements on the field $\boldsymbol{v}$ to give a meaning to the quantity $\int_{F}\left(\boldsymbol{v} \cdot \boldsymbol{n}_{K}\right) \phi \mathrm{d} s$, where $\phi$ is a smooth function on $F$ (e.g., a polynomial function). It is well established that it is possible to give a weak meaning in $H^{-\frac{1}{2}}(\partial K)$ to the normal trace of $\boldsymbol{v}$ on $\partial K$ by means of an integration by parts formula if $\boldsymbol{v} \in \boldsymbol{H}(\operatorname{div} ; K):=\left\{\boldsymbol{v} \in \boldsymbol{L}^{2}(K) \mid \nabla \cdot \boldsymbol{v} \in L^{2}(K)\right\}$. In this situation, one can define the normal trace $\gamma_{\partial K}^{\mathrm{d}}(\boldsymbol{v}) \in H^{-\frac{1}{2}}(\partial K)$ by setting

$$
\left\langle\gamma_{\partial K}^{\mathrm{d}}(\boldsymbol{v}), \psi\right\rangle_{\partial K}:=\int_{K}(\boldsymbol{v} \cdot \nabla w(\psi)+(\nabla \cdot \boldsymbol{v}) w(\psi)) \mathrm{d} x,
$$

for all $\psi \in H^{\frac{1}{2}}(\partial K)$, where $w(\psi) \in H^{1}(K)$ is a lifting of $\psi$, i.e., $\gamma_{\partial K}^{\mathrm{g}}(w(\psi))=\psi$, and $\gamma_{\partial K}^{\mathrm{g}}: H^{1}(K) \rightarrow H^{\frac{1}{2}}(\partial K)$ is the trace map locally in $K$. Then, one has $\gamma_{\partial K}^{\mathrm{d}}(\boldsymbol{v})=$ $\boldsymbol{v}_{\mid \partial K} \cdot \boldsymbol{n}_{K}$ whenever $\boldsymbol{v}$ is smooth, e.g., if $\boldsymbol{v} \in \boldsymbol{H}(\operatorname{div} ; K) \cap \boldsymbol{C}^{0}(\bar{K})$. However, the above meaning is too weak for our purpose because we need to localize the action of the normal trace to functions $\phi$ only defined on a face $F$, i.e., $\phi$ may not be defined over the whole boundary $\partial K$. The key to achieve this is to extend $\phi$ by zero from $F$ to $\partial K$. This obliges us to change the functional setting since the extended function is no longer in $H^{\frac{1}{2}}(\partial K)$. In what follows, we are going to use that the zero-extension of a smooth function defined on a face $F$ of $\partial K$ is in $W^{1-\frac{1}{t}, t}(\partial K)$ if $t \in[1,2)$, i.e., $t\left(1-\frac{1}{t}\right)<1$. Let us now present a rigorous construction.

Let $p, q$ be two real numbers such that

$$
p>2, \quad q>\frac{2 d}{2+d} .
$$

Notice that $q>1$ since $d \geq 2$. Let $\varrho \in(2, p]$ be such that $q \geq \frac{\varrho d}{\varrho+d}$; this is indeed possible since $p>2, q>\frac{2 d}{2+d}$, and the function $z \mapsto \frac{z d}{z+d}$ is increasing over $\mathbb{R}_{+}$. Lemma 3.1 shows that there exists a bounded lifting operator

$$
L_{F}^{K}: W^{\frac{1}{\varrho}, \varrho^{\prime}}(F) \longrightarrow W^{1, \varrho^{\prime}}(K),
$$

with conjugate number $\varrho^{\prime}$ s.t. $\frac{1}{\varrho}+\frac{1}{\varrho^{\prime}}=1$, so that for any $\phi \in W^{\frac{1}{\varrho}, \varrho^{\prime}}(F), L_{F}^{K}(\phi)$ is a lifting of the zero-extension of $\phi$ to $\partial K$, i.e.,

$$
\gamma_{\partial K}^{\mathrm{g}}\left(L_{F}^{K}(\phi)\right)_{\mid \partial K \backslash F}=0, \quad \gamma_{\partial K}^{\mathrm{g}}\left(L_{F}^{K}(\phi)\right)_{\mid F}=\phi .
$$

Notice that the domain of $L_{F}^{K}$ is of the form $W^{1-\frac{1}{t}, t}(F)$ with $t:=\varrho^{\prime}<2$, which is consistent with the above observation regarding the zero-extension to $\partial K$ of functions defined on $F$. We also observe that

$$
L_{F}^{K}(\phi) \in W^{1, p^{\prime}}(K) \cap L^{q^{\prime}}(K),
$$

with conjugate numbers $p^{\prime}, q^{\prime}$ s.t. $\frac{1}{p}+\frac{1}{p^{\prime}}=1, \frac{1}{q}+\frac{1}{q^{\prime}}=1$. Indeed, $L_{F}^{K}(\phi) \in W^{1, p^{\prime}}(K)$ just follows from $p^{\prime} \leq \varrho^{\prime}$ (i.e., $\varrho \leq p$ ), whereas $L_{F}^{K}(\phi) \in L^{q^{\prime}}(K)$ follows from $W^{1, \varrho^{\prime}}(K) \hookrightarrow L^{q^{\prime}}(K)$ owing to the Sobolev embedding theorem (since $q^{\prime} \leq \frac{\varrho^{\prime} d}{d-\varrho^{\prime}}$, as can be verified from $d \geq 2>\varrho^{\prime}$ and $\frac{1}{\varrho^{\prime}}-\frac{1}{d}=1-\left(\frac{1}{\varrho}+\frac{1}{d}\right) \leq 1-\frac{1}{q}=\frac{1}{q^{\prime}}$ because $\left.q \geq \frac{\varrho d}{\varrho+d}\right)$. We now state our main result on the lifting operator $L_{F}^{K}$. 
Lemma 3.1 (Face-to-cell lifting). Let $p$ and $q$ satisfy (3.2). Let $\varrho \in(2, p]$ be such that $q \geq \frac{\varrho d}{\varrho+d}$. For all $K \in \mathcal{T}_{h}$, and all $F \in \mathcal{F}_{K}$, there exists a lifting operator $L_{F}^{K}: W^{\frac{1}{\varrho}, \varrho^{\prime}}(F) \rightarrow W^{1, \varrho^{\prime}}(K)$ satisfying (3.4). Moreover, there exists $c$ so that for all $h \in \mathcal{H}$, all $K \in \mathcal{T}_{h}$, and all $F \in \mathcal{F}_{K}$, the following bound holds true:

$$
h_{K}^{\frac{d}{p}}\left|L_{F}^{K}(\phi)\right|_{W^{1, p^{\prime}}(K)}+h_{K}^{-1+\frac{d}{q}}\left\|L_{F}^{K}(\phi)\right\|_{L^{q^{\prime}}(K)} \leq c h_{K}^{-\frac{1}{\varrho}+\frac{d}{\varrho}}\|\phi\|_{W^{\frac{1}{\varrho}, \varrho^{\prime}}(F)},
$$

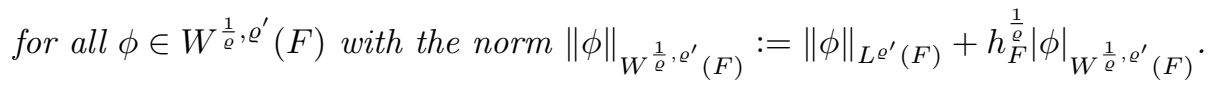

Proof. (1) The face-to-cell lifting operator $L_{F}^{K}$ is constructed from a lifting operator $L_{\widehat{F}}^{\widehat{K}}$ on the reference cell. Let $\widehat{K}$ be the reference cell and let $\widehat{F}$ be one of its faces. Let us define the operator $L_{\widehat{F}}^{\widehat{K}}: W^{\frac{1}{\varrho}, \varrho^{\prime}}(\widehat{F}) \rightarrow W^{1, \varrho^{\prime}}(\widehat{K})$. For any function $\psi \in W^{\frac{1}{\varrho}, \varrho^{\prime}}(\widehat{F})$, let $\widetilde{\psi}$ denote the zero-extension of $\psi$ to $\partial \widehat{K}$. Owing to Grisvard [28, Thm. 1.4.2.4, Cor. 1.4.4.5], $\widetilde{\psi}$ is in $W^{\frac{1}{\varrho}, \varrho^{\prime}}(\partial \widehat{K})$ since $\frac{\varrho^{\prime}}{\varrho}=\frac{1}{\varrho-1}<1$ (i.e., $\varrho>2$ ), and we have $\|\widetilde{\psi}\|_{W^{\frac{1}{\varrho}, \varrho^{\prime}}(\partial \widehat{K})} \leq \widehat{c}_{1}\|\psi\|_{W^{\frac{1}{\varrho}, \varrho^{\prime}}(\widehat{F})}$ with the norm $\|\psi\|_{W^{\frac{1}{\varrho}, \varrho^{\prime}}(\widehat{F})}:=$ $\|\psi\|_{L^{\varrho^{\prime}(\widehat{F})}}+\ell_{\widehat{K}}^{\frac{1}{\varrho}}|\psi|_{W^{\frac{1}{\varrho}, \varrho^{\prime}}(\widehat{F})}$ where $\ell_{\widehat{K}}=1$ is a length scale associated with $\widehat{K}$. Then we use the surjectivity of the trace map $\gamma_{\widehat{K}}^{\mathrm{g}}: W^{1, \varrho^{\prime}}(\widehat{K}) \rightarrow W^{\frac{1}{\varrho}, \varrho^{\prime}}(\partial \widehat{K})$ (see Gagliardo [27, Thm. 1.I] $)$ to define $L_{\widehat{F}}^{\widehat{K}}(\psi) \in W^{1, \varrho^{\prime}}(\widehat{K})$ s.t. $\gamma_{\widehat{K}}^{\mathrm{g}}\left(L_{\widehat{F}}^{\widehat{K}}(\psi)\right)=\widetilde{\psi}$ and $\left\|L_{\widehat{F}}^{\widehat{K}}(\psi)\right\|_{W^{1, \varrho^{\prime}}(\widehat{K})} \leq$ $\widehat{c}_{2}\|\widetilde{\psi}\|_{W^{\frac{1}{\varrho}, \varrho^{\prime}}(\partial \widehat{K})}$, i.e., $\left\|L_{\widehat{F}}^{\widehat{K}}(\psi)\right\|_{W^{1, \varrho^{\prime}}(\widehat{K})} \leq \widehat{c}\|\psi\|_{W^{\frac{1}{\varrho}, \varrho^{\prime}}(\widehat{F})}$, with $\widehat{c}=\widehat{c}_{1} \widehat{c}_{2}$. By construction, we have $\gamma_{\partial \widehat{K}}^{\mathrm{g}}\left(L_{\widehat{F}}^{\widehat{K}}(\psi)\right)_{\mid \widehat{F}}=\psi$ and $\gamma_{\partial \widehat{K}}^{\mathrm{g}}\left(L_{\widehat{F}}^{\widehat{K}}(\psi)\right)_{\mid \partial \widehat{K} \backslash \widehat{F}}=0$.

(2) We define the lifting operator $L_{F}^{K}: W^{\frac{1}{\varrho}, \varrho^{\prime}}(F) \rightarrow W^{1, \varrho^{\prime}}(K)$ by setting

$$
L_{F}^{K}(\phi)(\boldsymbol{x}):=L_{\widehat{F}}^{\widehat{K}}\left(\phi \circ \boldsymbol{T}_{K \mid \widehat{F}}\right)\left(\boldsymbol{T}_{K}^{-1}(\boldsymbol{x})\right), \quad \forall \boldsymbol{x} \in K, \quad \forall \phi \in W^{\frac{1}{\varrho}, \varrho^{\prime}}(F),
$$

where $\boldsymbol{T}_{K}: \widehat{K} \rightarrow K$ is the geometric mapping and $\widehat{F}=\boldsymbol{T}_{K}^{-1}(F)$. By definition, if $\boldsymbol{x} \in F$, then $\widehat{\boldsymbol{x}}:=\boldsymbol{T}_{K}^{-1}(\boldsymbol{x}) \in \widehat{F}$ and $\boldsymbol{T}_{K \mid \widehat{F}}(\widehat{\boldsymbol{x}})=\boldsymbol{x}$, so that

$$
\gamma_{\partial K}^{\mathrm{g}}\left(L_{F}^{K}(\phi)\right)(\boldsymbol{x})=\gamma_{\partial \widehat{K}}^{\mathrm{g}}\left(L_{\widehat{F}}^{\widehat{K}}\left(\phi \circ \boldsymbol{T}_{K \mid \widehat{F}}\right)\right)(\widehat{\boldsymbol{x}})=\phi\left(\boldsymbol{T}_{K \mid \widehat{F}}(\widehat{\boldsymbol{x}})\right)=\phi(\boldsymbol{x}),
$$

whereas if $\boldsymbol{x} \in \partial K \backslash F$, then $\widehat{\boldsymbol{x}} \in \partial \widehat{K} \backslash \widehat{F}$, so that $\gamma_{\partial \widehat{K}}^{\mathrm{g}}\left(L_{\widehat{F}}^{\widehat{K}}\left(\phi \circ \boldsymbol{T}_{K \mid \widehat{F}}\right)\right)(\widehat{\boldsymbol{x}})=0$. The above argument shows that (3.4) holds true.

(3) It remains to prove (3.6). Let us first bound $\left|L_{F}^{K}(\phi)\right|_{W^{1, p^{\prime}}(K)}$. Notice that the definition of $L_{K}^{F}$ is equivalent to $L_{F}^{K}(\phi) \circ \boldsymbol{T}_{K}(\widehat{\boldsymbol{x}}):=L_{\widehat{F}}^{\widehat{K}}\left(\phi \circ \boldsymbol{T}_{K \mid \widehat{F}}\right)(\widehat{\boldsymbol{x}})$; that is, $\psi_{K}^{\mathrm{g}}\left(L_{F}^{K}(\phi)\right):=L_{\widehat{F}}^{\widehat{K}}\left(\psi_{F}^{\mathrm{g}}(\phi)\right)$, where $\psi_{K}^{\mathrm{g}}$ is the pullback by $\boldsymbol{T}_{K}$, and $\psi_{F}^{\mathrm{g}}$ is the pullback by $\boldsymbol{T}_{K \mid \widehat{F}}$. Denoting by $\mathbb{J}_{K}$ the Jacobian of the geometric mapping $\boldsymbol{T}_{K}$, we infer that

$$
\begin{aligned}
\left|L_{F}^{K}(\phi)\right|_{W^{1, p^{\prime}}(K)} & \leq c\left\|\mathbb{J}_{K}^{-1}\right\|_{\ell^{2}}\left|\operatorname{det}\left(\mathbb{J}_{K}\right)\right|^{\frac{1}{p^{\prime}}}\left|L_{\widehat{K}}^{\widehat{K}}\left(\psi_{F}^{\mathrm{g}}(\phi)\right)\right|_{W^{1, p^{\prime}}(\widehat{K})} \\
& \leq c^{\prime}\left\|\mathbb{J}_{K}^{-1}\right\|_{\ell^{2}}\left|\operatorname{det}\left(\mathbb{J}_{K}\right)\right|^{\frac{1}{p^{\prime}}}\left|L_{\widehat{F}}^{\widehat{K}}\left(\psi_{F}^{\mathrm{g}}(\phi)\right)\right|_{W^{1, \varrho^{\prime}}(\widehat{K})} \\
& \leq c^{\prime \prime}\left\|\mathbb{J}_{K}^{-1}\right\|_{\ell^{2}}\left|\operatorname{det}\left(\mathbb{J}_{K}\right)\right|^{\frac{1}{p^{\prime}}}\left\|\psi_{F}^{\mathrm{g}}(\phi)\right\|_{W^{\frac{1}{\varrho}, \varrho^{\prime}}(\widehat{F})},
\end{aligned}
$$

where the first inequality follows from the chain rule, the second is a consequence of $\varrho^{\prime} \geq p^{\prime}$ ( since $\varrho \leq p$ ), and the third follows from the stability of the reference lifting 
operator $L_{\widehat{F}}^{\widehat{K}}$. Using now the chain rule and the shape-regularity of the mesh sequence, we infer that $\left\|\psi_{F}^{\mathrm{g}}(\phi)\right\|_{W^{\frac{1}{\varrho}, \varrho^{\prime}}(\widehat{F})} \leq c\left|\operatorname{det}\left(\mathbb{J}_{F}\right)\right|^{-\frac{1}{\varrho^{\prime}}}\|\phi\|_{W^{\frac{1}{\varrho}, \varrho^{\prime}}(F)}$, where $\mathbb{J}_{F}$ is the Jacobian of the mapping $\boldsymbol{T}_{K \mid \widehat{F}}: \widehat{F} \rightarrow F$. Combining these bounds, we obtain

$$
\begin{aligned}
\left|L_{F}^{K}(\phi)\right|_{W^{1, p^{\prime}}(K)} & \leq c\left\|\mathbb{J}_{K}^{-1}\right\|_{\ell^{2}}\left|\operatorname{det}\left(\mathbb{J}_{K}\right)\right|^{\frac{1}{p^{\prime}}}\left|\operatorname{det}\left(\mathbb{J}_{F}\right)\right|^{-\frac{1}{\varrho^{\prime}}}\|\phi\|_{W^{\frac{1}{\varrho}, \varrho^{\prime}}(F)} \\
& \leq c^{\prime} h_{K}^{-\frac{1}{\varrho}+d\left(\frac{1}{\varrho}-\frac{1}{p}\right)}\|\phi\|_{W^{\frac{1}{\varrho}, \varrho^{\prime}}(F)},
\end{aligned}
$$

where the second bound follows from the shape-regularity of the mesh sequence. This proves the bound on $\left|L_{F}^{K}(\phi)\right|_{W^{1, p^{\prime}}(K)}$ in (3.6). The proof of the bound on $\left\|L_{F}^{K}(\phi)\right\|_{L^{q^{\prime}(K)}}$ uses similar arguments together with $W^{1, \varrho^{\prime}}(\widehat{K}) \hookrightarrow L^{q^{\prime}}(\widehat{K})$ owing to the Sobolev embedding theorem and $q^{\prime} \leq \frac{\varrho^{\prime} d}{d-\varrho^{\prime}}$ (as already shown above).

3.2. Face localization of the normal diffusive flux. Let $K \in \mathcal{T}_{h}$ be a mesh cell, $F \in \mathcal{F}_{K}$ be a face of $K$, and consider the following functional space:

$$
\boldsymbol{S}^{\mathrm{d}}(K):=\left\{\boldsymbol{\tau} \in \boldsymbol{L}^{p}(K) \mid \nabla \cdot \boldsymbol{\tau} \in L^{q}(K)\right\},
$$

equipped with the following dimensionally-consistent norm:

$$
\|\boldsymbol{\tau}\|_{S^{\mathrm{d}}(K)}:=\|\boldsymbol{\tau}\|_{\boldsymbol{L}^{p}(K)}+h_{K}^{1+d\left(\frac{1}{p}-\frac{1}{q}\right)}\|\nabla \cdot \boldsymbol{\tau}\|_{L^{q}(K)} .
$$

With the lifting operator $L_{F}^{K}$ in hand, we now define the normal trace on the face $F$ of $K$ of any field $\boldsymbol{\tau} \in \boldsymbol{S}^{\mathrm{d}}(K)$ to be the linear form in $\left(W^{\frac{1}{\varrho}, \varrho^{\prime}}(F)\right)^{\prime}$ denoted by $\left(\boldsymbol{\tau} \cdot \boldsymbol{n}_{K}\right)_{\mid F}$ and whose action on any function $\phi \in W^{\frac{1}{\varrho}, \varrho^{\prime}}(F)$ is

$$
\left\langle\left(\boldsymbol{\tau} \cdot \boldsymbol{n}_{K}\right)_{\mid F}, \phi\right\rangle_{F}:=\int_{K}\left(\boldsymbol{\tau} \cdot \nabla L_{F}^{K}(\phi)+(\nabla \cdot \boldsymbol{\tau}) L_{F}^{K}(\phi)\right) \mathrm{d} x .
$$

Here, $\langle\cdot, \cdot\rangle_{F}$ denotes the duality pairing between $\left(W^{\frac{1}{\varrho}, \varrho^{\prime}}(F)\right)^{\prime}$ and $W^{\frac{1}{\varrho}, \varrho^{\prime}}(F)$. Notice that the right-hand side of (3.10) is well-defined owing to Hölder's inequality and (3.6). Owing to (3.4), we readily verify that we have indeed defined an extension of the normal trace since we have $\left\langle\left(\boldsymbol{\tau} \cdot \boldsymbol{n}_{K}\right)_{\mid F}, \phi\right\rangle_{F}=\int_{F}\left(\boldsymbol{\tau} \cdot \boldsymbol{n}_{K}\right) \phi \mathrm{d} s$ whenever the field $\boldsymbol{\tau}$ is smooth. Let us now derive an important bound on the linear form $\left(\boldsymbol{\tau} \cdot \boldsymbol{n}_{K}\right)_{\mid F}$ when it acts on a function from the space $P_{F}$, which we define to be composed of the restrictions to $F$ of the functions in $P_{K}$. Note that $P_{F} \subset W^{\frac{1}{\varrho}, \varrho^{\prime}}(F)$.

Lemma 3.2 (Bound on normal component). Let $p$ and $q$ satisfy (3.2). There exists $c$ so that the following holds true:

$$
\left|\left\langle\left(\boldsymbol{\tau} \cdot \boldsymbol{n}_{K}\right)_{\mid F}, \phi_{h}\right\rangle_{F}\right| \leq c h_{K}^{d\left(\frac{1}{2}-\frac{1}{p}\right)}\|\boldsymbol{\tau}\|_{S^{\mathrm{d}}(K)} h_{F}^{-\frac{1}{2}}\left\|\phi_{h}\right\|_{L^{2}(F)},
$$

for all $\boldsymbol{\tau} \in \boldsymbol{S}^{\mathrm{d}}(K)$, all $\phi_{h} \in P_{F}$, all $K \in \mathcal{T}_{h}$, all $F \in \mathcal{F}_{K}$, and all $h \in \mathcal{H}$.

Proof. A direct consequence of (3.10), Hölder's inequality, and Lemma 3.1 is that

$$
\left|\left\langle\left(\boldsymbol{\tau} \cdot \boldsymbol{n}_{K}\right)_{\mid F}, \phi\right\rangle_{F}\right| \leq c h_{K}^{-\frac{1}{\varrho}+d\left(\frac{1}{\varrho}-\frac{1}{p}\right)}\|\boldsymbol{\tau}\|_{S^{\mathrm{d}}(K)}\|\phi\|_{W^{\frac{1}{\varrho}, \varrho^{\prime}}(F)},
$$

for all $\phi \in W^{\frac{1}{\varrho}, \varrho^{\prime}}(F)$. Recalling that $\|\phi\|_{W^{\frac{1}{\varrho}, \varrho^{\prime}}(F)}=\|\phi\|_{L^{\varrho^{\prime}(F)}}+h_{F}^{\frac{1}{\varrho}}|\phi|_{W^{\frac{1}{\varrho}, \varrho^{\prime}}(F)}$, the shape-regularity of the mesh sequence implies that the following inverse inequality $\left\|\phi_{h}\right\|_{W^{\frac{1}{\varrho}, \varrho^{\prime}}(F)} \leq c h_{F}^{(d-1)\left(\frac{1}{2}-\frac{1}{\varrho}\right)}\left\|\phi_{h}\right\|_{L^{2}(F)}$ holds true for all $\phi_{h} \in P_{F}$ (note that $\frac{1}{2}-\frac{1}{\varrho}=$ $\left.\frac{1}{\varrho^{\prime}}-\frac{1}{2}\right)$. The estimate (3.11) follows readily. 
3.3. Definition of $n_{\sharp}$ and key identities. Let us consider the functional space $V_{\mathrm{S}}$ defined in (2.5), where we recall that the real numbers $p$ and $q$ involved in the definition of $V_{\mathrm{S}}$ satisfy (3.2) and $q \leq 2$. For all $v \in V_{\mathrm{S}}$, Lemma 2.2 shows that $\boldsymbol{\sigma}(v)_{\mid K} \in \boldsymbol{S}^{\mathrm{d}}(K)$ for all $K \in \mathcal{T}_{h}$, and Lemma 3.2 implies that it is possible to give a meaning by duality to the normal component of $\boldsymbol{\sigma}(v)_{\mid K}$ on all the faces of $K$ separately. Moreover, since we have set $\boldsymbol{\sigma}\left(v_{h}\right)_{\mid K}:=-\lambda_{K} \nabla\left(v_{h \mid K}\right)$ for all $v_{h} \in P_{k}^{\mathrm{b}}\left(\mathcal{T}_{h}\right)$, and since we have $P_{K} \subset W^{k+1, \infty}(K)$ with $k \geq 1$, we infer that $\boldsymbol{\sigma}\left(v_{h}\right)_{\mid K} \in \boldsymbol{S}^{\mathrm{d}}(K)$ as well. Thus, $\boldsymbol{\sigma}(v)_{\mid K} \in \boldsymbol{S}^{\mathrm{d}}(K)$ for all $v \in\left(V_{\mathrm{S}}+P_{k}^{\mathrm{b}}\left(\mathcal{T}_{h}\right)\right)$. Let us now introduce the bilinear form $n_{\sharp}:\left(V_{\mathrm{S}}+P_{k}^{\mathrm{b}}\left(\mathcal{T}_{h}\right)\right) \times P_{k}^{\mathrm{b}}\left(\mathcal{T}_{h}\right) \rightarrow \mathbb{R}$ defined as follows:

$$
n_{\sharp}\left(v, w_{h}\right):=\sum_{F \in \mathcal{F}_{h}} \sum_{K \in \mathcal{T}_{F}} \epsilon_{K, F} \theta_{K, F}\left\langle\left(\boldsymbol{\sigma}(v)_{\mid K} \cdot \boldsymbol{n}_{K}\right)_{\mid F}, \llbracket w_{h} \rrbracket\right\rangle_{F},
$$

where the weights $\theta_{K, F}$ are still unspecified but are assumed to satisfy

$$
\theta_{K_{l}, F}, \theta_{K_{r}, F} \in[0,1] \text { and } \theta_{K_{l}, F}+\theta_{K_{r}, F}=1, \quad \forall F \in \mathcal{F}_{h}^{\circ},
$$

whereas for all $F \in \mathcal{F}_{h}^{\partial}$ with $F=\partial K_{l} \cap \partial D$, we set $\theta_{K_{l}, F}:=1, \theta_{K_{r}, F}=: 0$. We will see in (3.19) below how these weights must depend on the diffusion coefficient to get a robust boundedness estimate on $n_{\sharp}$. The definition (3.12) is meaningful since $\llbracket w_{h} \rrbracket_{F} \in P_{F} \subset W^{\frac{1}{\varrho}, \varrho^{\prime}}(F)$ for all $w_{h} \in P_{k}^{\mathrm{b}}\left(\mathcal{T}_{h}\right)$. The purpose of the factor $\epsilon_{K, F}=\boldsymbol{n}_{F} \cdot \boldsymbol{n}_{K \mid F}$ in (3.12) is to handle the relative orientation of $\boldsymbol{n}_{K}$ and $\boldsymbol{n}_{F}$. For all $v \in W^{1,1}\left(\mathcal{T}_{h}\right)$, we define weighted averages as follows for a.e. $\boldsymbol{x} \in F \in \mathcal{F}_{h}^{\circ}$ :

$$
\begin{aligned}
& \{v\}_{F, \theta}(\boldsymbol{x}):=\theta_{K_{l}, F} v_{\mid K_{l}}(\boldsymbol{x})+\theta_{K_{r}, F} v_{\mid K_{r}}(\boldsymbol{x}), \\
& \{v\}_{F, \bar{\theta}}(\boldsymbol{x}):=\theta_{K_{r}, F} v_{\mid K_{l}}(\boldsymbol{x})+\theta_{K_{l}, F} v_{\mid K_{r}}(\boldsymbol{x}) .
\end{aligned}
$$

Whenever $\theta_{K_{l}, F}=\theta_{K_{r}, F}=\frac{1}{2}$, these two definitions coincide with the usual arithmetic average. On boundary faces $F \in \mathcal{F}_{h}^{\partial}$, we set $\{v\}_{F, \theta}(\boldsymbol{x}):=v_{\mid K_{l}}(\boldsymbol{x})$, and $\{v\}_{F, \bar{\theta}}(\boldsymbol{x}):=0$ for a.e. $\boldsymbol{x} \in F$. We omit the subscript ${ }_{F}$ whenever the context is unambiguous. The following identity will be useful:

$$
\llbracket v w \rrbracket=\{v\}_{\theta} \llbracket w \rrbracket+\llbracket v \rrbracket\{w\}_{\bar{\theta}} .
$$

The following lemma is fundamental to understand the role that the bilinear form $n_{\sharp}$ will play in the next section in the analysis of various nonconforming approximation methods.

LEMma 3.3 (Identities for $n_{\sharp}$ ). The following holds true for any choice of weights $\left\{\theta_{K, F}\right\}_{F \in \mathcal{F}_{h}, K \in \mathcal{T}_{F}}$ and for all $w_{h} \in P_{k}^{\mathrm{b}}\left(\mathcal{T}_{h}\right)$, all $v_{h} \in P_{k}^{\mathrm{b}}\left(\mathcal{T}_{h}\right)$, and all $v \in V_{\mathrm{S}}$ :

$$
\begin{aligned}
n_{\sharp}\left(v_{h}, w_{h}\right) & =\sum_{F \in \mathcal{F}_{h}} \int_{F}\left\{\boldsymbol{\sigma}\left(v_{h}\right)\right\}_{\theta} \cdot \boldsymbol{n}_{F} \llbracket w_{h} \rrbracket \mathrm{d} s, \\
n_{\sharp}\left(v, w_{h}\right) & =\sum_{K \in \mathcal{T}_{h}} \int_{K}\left(\boldsymbol{\sigma}(v) \cdot \nabla w_{h \mid K}+(\nabla \cdot \boldsymbol{\sigma}(v)) w_{h \mid K}\right) \mathrm{d} x .
\end{aligned}
$$

Proof. (1) Proof of (3.16a). Let $v_{h}, w_{h} \in P_{k}^{\mathrm{b}}\left(\mathcal{T}_{h}\right)$. Since the restriction of $\boldsymbol{\sigma}\left(v_{h}\right)$ to each mesh cell is smooth, and since the restriction of $L_{F}^{K}\left(\llbracket w_{h} \rrbracket\right)$ to $\partial K$ is nonzero only on the face $F \in \mathcal{F}_{K}$ where it coincides with $\llbracket w_{h} \rrbracket$, we have

$$
\begin{aligned}
\left\langle\left(\boldsymbol{\sigma}\left(v_{h}\right)_{\mid K} \cdot \boldsymbol{n}_{K}\right)_{\mid F}, \llbracket w_{h} \rrbracket\right\rangle_{F} & =\int_{K}\left(\boldsymbol{\sigma}\left(v_{h}\right)_{\mid K} \cdot \nabla L_{F}^{K}\left(\llbracket w_{h} \rrbracket\right)+\left(\nabla \cdot \boldsymbol{\sigma}\left(v_{h}\right)_{\mid K}\right) L_{F}^{K}\left(\llbracket w_{h} \rrbracket\right)\right) \mathrm{d} x \\
& =\int_{\partial K} \boldsymbol{\sigma}\left(v_{h}\right)_{\mid K} \cdot \boldsymbol{n}_{K} L_{F}^{K}\left(\llbracket w_{h} \rrbracket\right) \mathrm{d} s=\int_{F} \boldsymbol{\sigma}\left(v_{h}\right)_{\mid K} \cdot \boldsymbol{n}_{K} \llbracket w_{h} \rrbracket \mathrm{d} s,
\end{aligned}
$$


where we used the divergence formula in $K$. Therefore, after using the definitions of $\epsilon_{K, F}$ and of $\theta_{K, F}$, we obtain

$$
\begin{aligned}
n_{\sharp}\left(v_{h}, w_{h}\right) & =\sum_{F \in \mathcal{F}_{h}} \sum_{K \in \mathcal{T}_{F}} \epsilon_{K, F} \theta_{K, F} \int_{F} \boldsymbol{\sigma}\left(v_{h}\right)_{\mid K} \cdot \boldsymbol{n}_{K} \llbracket w_{h} \rrbracket \mathrm{d} s \\
& =\sum_{F \in \mathcal{F}_{h}} \int_{F}\left\{\boldsymbol{\sigma}\left(v_{h}\right)\right\}_{\theta} \cdot \boldsymbol{n}_{F} \llbracket w_{h} \rrbracket \mathrm{d} s .
\end{aligned}
$$

(2) Proof of (3.16b). Let $v \in V_{\mathrm{S}}$ and $w_{h} \in P_{k}^{\mathrm{b}}\left(\mathcal{T}_{h}\right)$. Let $\mathcal{K}_{\delta}^{\mathrm{d}}: \boldsymbol{L}^{1}(D) \rightarrow \boldsymbol{C}^{\infty}(\bar{D})$ and $\mathcal{K}_{\delta}^{\mathrm{b}}: L^{1}(D) \rightarrow C^{\infty}(\bar{D})$ be the mollification operators introduced in [22, §3.2]. These two operators satisfy the following key commuting property:

$$
\nabla \cdot\left(\mathcal{K}_{\delta}^{\mathrm{d}}(\boldsymbol{\tau})\right)=\mathcal{K}_{\delta}^{\mathrm{b}}(\nabla \cdot \boldsymbol{\tau})
$$

for all $\boldsymbol{\tau} \in \boldsymbol{L}^{1}(D)$ s.t. $\nabla \cdot \boldsymbol{\tau} \in L^{1}(D)$. It is important to realize that this property can be applied to $\boldsymbol{\sigma}(v)$ for all $v \in V_{\mathrm{S}}$ since $\nabla \cdot \boldsymbol{\sigma}(v) \in L^{1}(D)$ by definition of $V_{\mathrm{S}}$. (Note that this property cannot be applied to $\sigma\left(v_{h}\right)$ with $v_{h} \in P_{k}^{\mathrm{b}}\left(\mathcal{T}_{h}\right)$, since the normal component of $\boldsymbol{\sigma}\left(v_{h}\right)$ is in general discontinuous across the mesh interfaces, i.e., $\boldsymbol{\sigma}\left(v_{h}\right)$ does not have a weak divergence.) Let us consider the mollified bilinear form

$$
n_{\sharp \delta}\left(v, w_{h}\right):=\sum_{F \in \mathcal{F}_{h}} \sum_{K \in \mathcal{T}_{F}} \epsilon_{K, F} \theta_{K, F}\left\langle\left(\mathcal{K}_{\delta}^{\mathrm{d}}(\boldsymbol{\sigma}(v))_{\mid K} \cdot \boldsymbol{n}_{K}\right)_{\mid F}, \llbracket w_{h} \rrbracket\right\rangle_{F} .
$$

Owing to the commuting property (3.17), we infer that

$$
\begin{aligned}
& \left\langle\left(\mathcal{K}_{\delta}^{\mathrm{d}}(\boldsymbol{\sigma}(v))_{\mid K} \cdot \boldsymbol{n}_{K}\right)_{\mid F}, \llbracket w_{h} \rrbracket\right\rangle_{F}= \\
& \int_{K}\left(\mathcal{K}_{\delta}^{\mathrm{d}}(\boldsymbol{\sigma}(v)) \cdot L_{F}^{K}\left(\llbracket w_{h} \rrbracket\right)+\mathcal{K}_{\delta}^{\mathrm{b}}(\nabla \cdot \boldsymbol{\sigma}(v)) L_{F}^{K}\left(\llbracket w_{h} \rrbracket\right)\right) \mathrm{d} x .
\end{aligned}
$$

Then Theorem 3.3 from [22] implies that

$$
\begin{aligned}
& \lim _{\delta \rightarrow 0} \int_{K}\left(\mathcal{K}_{\delta}^{\mathrm{d}}(\boldsymbol{\sigma}(v)) \cdot L_{F}^{K}\left(\llbracket w_{h} \rrbracket\right)+\mathcal{K}_{\delta}^{\mathrm{b}}((\nabla \cdot \boldsymbol{\sigma}(v))) L_{F}^{K}\left(\llbracket w_{h} \rrbracket\right)\right) \mathrm{d} x= \\
& \int_{K}\left(\boldsymbol{\sigma}(v) \cdot L_{F}^{K}\left(\llbracket w_{h} \rrbracket\right)+(\nabla \cdot \boldsymbol{\sigma}(v)) L_{F}^{K}\left(\llbracket w_{h} \rrbracket\right)\right) \mathrm{d} x=\left\langle\left(\boldsymbol{\sigma}(v)_{\mid K} \cdot \boldsymbol{n}_{K}\right)_{\mid F}, \llbracket w_{h} \rrbracket\right\rangle_{F} .
\end{aligned}
$$

Summing over the mesh faces and the associated mesh cells, we infer that

$$
\lim _{\delta \rightarrow 0} n_{\sharp \delta}\left(v, w_{h}\right)=n_{\sharp}\left(v, w_{h}\right) .
$$

Moreover, since the mollified function $\mathcal{K}_{\delta}^{\mathrm{d}}(\boldsymbol{\sigma}(v))$ is smooth, by repeating the calculation done in Step (1), we also have

$$
n_{\sharp \delta}\left(v, w_{h}\right)=\sum_{F \in \mathcal{F}_{h}} \int_{F}\left\{\mathcal{K}_{\delta}^{\mathrm{d}}(\boldsymbol{\sigma}(v))\right\}_{\theta} \cdot \boldsymbol{n}_{F} \llbracket w_{h} \rrbracket \mathrm{d} s .
$$

Using the identity (3.15) with $\llbracket \mathcal{K}_{\delta}^{\mathrm{d}}(\boldsymbol{\sigma}(v)) \rrbracket \cdot \boldsymbol{n}_{F}=0$ for all $F \in \mathcal{F}_{h}^{\circ}$, recalling that $\llbracket w_{h} \mathcal{K}_{\delta}^{\mathrm{d}}(\boldsymbol{\sigma}(v)) \rrbracket=w_{h} \mathcal{K}_{\delta}^{\mathrm{d}}(\boldsymbol{\sigma}(v))_{\mid F}$ for all $F \in \mathcal{F}_{h}^{\partial}$, and using the divergence formula in 
$K$ and the commuting property (3.17), we obtain

$$
\begin{aligned}
n_{\sharp \delta}\left(v, w_{h}\right) & =\sum_{F \in \mathcal{F}_{h}} \int_{F}\left\{\mathcal{K}_{\delta}^{\mathrm{d}}(\boldsymbol{\sigma}(v))\right\}_{\theta} \cdot \boldsymbol{n}_{F} \llbracket w_{h} \rrbracket \mathrm{d} s+\sum_{F \in \mathcal{F}_{h}^{\circ}} \int_{F} \llbracket \mathcal{K}_{\delta}^{\mathrm{d}}(\boldsymbol{\sigma}(v)) \rrbracket \cdot \boldsymbol{n}_{F}\left\{w_{h}\right\}_{\bar{\theta}} \mathrm{d} s \\
& =\sum_{F \in \mathcal{F}_{h}} \int_{F} \llbracket w_{h} \mathcal{K}_{\delta}^{\mathrm{d}}(\boldsymbol{\sigma}(v)) \rrbracket \cdot \boldsymbol{n}_{F} \mathrm{~d} s=\sum_{K \in \mathcal{T}_{h}} \int_{\partial K} \mathcal{K}_{\delta}^{\mathrm{d}}(\boldsymbol{\sigma}(v)) \cdot \boldsymbol{n}_{K} w_{h \mid K} \mathrm{~d} s \\
& =\sum_{K \in \mathcal{T}_{h}} \int_{K}\left(\mathcal{K}_{\delta}^{\mathrm{d}}(\boldsymbol{\sigma}(v)) \cdot \nabla w_{h \mid K}+\mathcal{K}_{\delta}^{\mathrm{b}}(\nabla \cdot \boldsymbol{\sigma}(v)) w_{h \mid K}\right) \mathrm{d} x .
\end{aligned}
$$

Invoking again Theorem 3.3 from [22] leads to the assertion since

$$
\lim _{\delta \rightarrow 0} n_{\sharp \delta}\left(v, w_{h}\right)=\sum_{K \in \mathcal{T}_{h}} \int_{K}\left(\boldsymbol{\sigma}(v) \cdot \nabla w_{h \mid K}+(\nabla \cdot \boldsymbol{\sigma}(v)) w_{h \mid K}\right) \mathrm{d} x .
$$

Remark 3.4 (Identity (3.16b)). The identity (3.16b) is the key tool to assert in a weak sense that $\boldsymbol{\sigma}(v) \cdot \boldsymbol{n}$ is continuous across the mesh interfaces without the need to assume that $v$ is smooth, say $v \in H^{1+r}(D)$ with $r>\frac{1}{2}$.

We now establish an important boundedness estimate on the bilinear form $n_{\sharp}$. Since $\boldsymbol{\sigma}(v)_{\mid K} \in \boldsymbol{S}^{\mathrm{d}}(K)$ for all $K \in \mathcal{T}_{h}$ and all $v \in V_{\mathrm{S}}+P_{k}^{\mathrm{b}}\left(\mathcal{T}_{h}\right)$, we can equip the space $V_{\mathrm{S}}+P_{k}^{\mathrm{b}}\left(\mathcal{T}_{h}\right)$ with the seminorm

$$
|v|_{n_{\sharp}}^{2}:=\sum_{K \in \mathcal{T}_{h}} \lambda_{K}^{-1}\left(h_{K}^{2 d\left(\frac{1}{2}-\frac{1}{p}\right)}\left\|\boldsymbol{\sigma}(v)_{\mid K}\right\|_{\boldsymbol{L}^{p}(K)}^{2}+h_{K}^{2 d\left(\frac{2+d}{2 d}-\frac{1}{q}\right)}\left\|\nabla \cdot \boldsymbol{\sigma}(v)_{\mid K}\right\|_{L^{q}(K)}^{2}\right) .
$$

We notice that this seminorm is dimensionally-consistent with the classical energynorm defined as $\sum_{K \in \mathcal{T}_{h}} \lambda_{K}\left\|\nabla v_{\mid K}\right\|_{L^{2}(K)}^{2}$. Straightforward algebra shows that $|v|_{n_{\sharp}} \leq$ $c \lambda_{b}^{-\frac{1}{2}}\left(\ell_{D}^{d\left(\frac{1}{2}-\frac{1}{p}\right)}\|\boldsymbol{\sigma}(v)\|_{\boldsymbol{L}^{p}(D)}+\ell_{D}^{d\left(\frac{2+d}{2 d}-\frac{1}{q}\right)}\|\nabla \cdot \boldsymbol{\sigma}(v)\|_{L^{q}(D)}\right)$, for all $v \in V_{\mathrm{S}}$; here $\ell_{D}$ denotes a characteristic length of $D$. (For the first term, use Hölder's inequality and $\sum_{K \in \mathcal{T}_{h}} h_{K}^{d} \leq c|\Omega|$, whereas for the second term, use that $h_{K} \leq \ell_{D}$ and $\|a\|_{\ell^{2}(\mathcal{I})} \leq$ $\|a\|_{\ell^{q}(\mathcal{I})}$ for any finite sequence $\left(a_{i}\right)_{i \in \mathcal{I}}$ since $q \leq 2$.)

In order to get robust error estimates, one should avoid any dependency on the ratio of the values taken by $\lambda$ in two adjacent subdomains. To avoid such dependencies, we introduce the following diffusion-dependent weights for all $F \in \mathcal{F}_{h}^{\circ}$ with $F=\partial K_{l} \cap \partial K_{r}$ :

$$
\theta_{K_{l}, F}:=\frac{\lambda_{K_{r}}}{\lambda_{K_{l}}+\lambda_{K_{r}}}, \quad \theta_{K_{r}, F}:=\frac{\lambda_{K_{l}}}{\lambda_{K_{l}}+\lambda_{K_{r}}} .
$$

We also define

$$
\lambda_{F}:=\frac{2 \lambda_{K_{l}} \lambda_{K_{r}}}{\lambda_{K_{l}}+\lambda_{K_{r}}} \text { if } F \in \mathcal{F}_{h}^{\circ} \quad \text { and } \quad \lambda_{F}:=\lambda_{K_{l}} \text { if } F \in \mathcal{F}_{h}^{\partial} .
$$

Recall that we have already defined $\theta_{K_{l}, F}:=1, \theta_{K_{r}, F}=: 0$ for all $F \in \mathcal{F}_{h}^{\partial}$. The two properties we are going to use are that $\left|\mathcal{T}_{F}\right| \lambda_{K} \theta_{K, F}=\lambda_{F}$ for all $K \in \mathcal{T}_{F}$, and $\lambda_{F} \leq \min _{K \in \mathcal{T}_{F}} \lambda_{K}$. (Here, $\left|\mathcal{T}_{F}\right|$ denotes the cardinality of $\mathcal{T}_{F}$.)

Lemma 3.5 (Boundedness of $n_{\sharp}$ ). Let the weights $\left\{\theta_{K, F}\right\}_{K \in \mathcal{T}_{h}}$ and the coefficients $\left\{\lambda_{F}\right\}_{F \in \mathcal{F}}$ be defined in (3.19) and (3.20). There is $c$ so that the following holds for all $h \in \mathcal{H}$, all $\lambda \in \Lambda(\Pi)$, all $v \in V_{\mathrm{S}}+P_{k}^{\mathrm{b}}\left(\mathcal{T}_{h}\right)$, and all $w_{h} \in P_{k}^{\mathrm{b}}\left(\mathcal{T}_{h}\right)$ :

$$
\left|n_{\sharp}\left(v, w_{h}\right)\right| \leq c|v|_{n_{\sharp}}\left(\sum_{F \in \mathcal{F}_{h}} \lambda_{F} h_{F}^{-1}\left\|\llbracket \llbracket w_{h} \rrbracket\right\|_{L^{2}(F)}^{2}\right)^{\frac{1}{2}} .
$$


Proof. Let $v \in V_{\mathrm{S}}+P_{k}^{\mathrm{b}}\left(\mathcal{T}_{h}\right)$ and $w_{h} \in P_{k}^{\mathrm{b}}\left(\mathcal{T}_{h}\right)$. Owing to the definition of $n_{\sharp}$ in (3.12) and the estimate (3.11) from Lemma 3.2, we infer that

$$
\begin{aligned}
& \left|n_{\sharp}\left(v, w_{h}\right)\right| \leq c \sum_{F \in \mathcal{F}_{h}} \sum_{K \in \mathcal{T}_{F}} \theta_{K, F} h_{K}^{d\left(\frac{1}{2}-\frac{1}{p}\right)}\left\|\boldsymbol{\sigma}(v)_{\mid K}\right\|_{S^{\mathrm{d}}(K)} h_{F}^{-\frac{1}{2}}\left\|\llbracket w_{h} \rrbracket\right\|_{L^{2}(F)} \\
& \leq c\left(\sum_{F \in \mathcal{F}_{h}} \sum_{K \in \mathcal{T}_{F}} \lambda_{K}^{-\frac{1}{2}} h_{K}^{d\left(\frac{1}{2}-\frac{1}{p}\right)}\left\|\boldsymbol{\sigma}(v)_{\mid K}\right\|_{\boldsymbol{L}^{p}(K)}\left|\mathcal{T}_{F}\right|^{-\frac{1}{2}} \lambda_{F}^{\frac{1}{2}} h_{F}^{-\frac{1}{2}}\left\|\llbracket w_{h} \rrbracket\right\|_{L^{2}(F)}\right. \\
& \left.+\sum_{F \in \mathcal{F}_{h}} \sum_{K \in \mathcal{T}_{F}} \lambda_{K}^{-\frac{1}{2}} h_{K}^{d\left(\frac{2+d}{2 d}-\frac{1}{q}\right)}\left\|\nabla \cdot \boldsymbol{\sigma}(v)_{\mid K}\right\|_{L^{q}(K)}\left|\mathcal{T}_{F}\right|^{-\frac{1}{2}} \lambda_{F}^{\frac{1}{2}} h_{F}^{-\frac{1}{2}}\left\|\llbracket w_{h} \rrbracket\right\|_{L^{2}(F)}\right),
\end{aligned}
$$

where we used that $\theta_{K, F} \leq \theta_{K, F}^{\frac{1}{2}}$ (since $\left.\theta_{K, F} \leq 1\right),\left|\mathcal{T}_{F}\right| \lambda_{K} \theta_{K, F}=\lambda_{F}$, the definition of $\|\cdot\|_{S^{\mathrm{d}}(K)}$, and $1+d\left(\frac{1}{2}-\frac{1}{q}\right)=d\left(\frac{2+d}{2 d}-\frac{1}{q}\right)$. Owing to the Cauchy-Schwarz inequality, we infer that $\sum_{F \in \mathcal{F}_{h}} \sum_{K \in \mathcal{T}_{F}} a_{K}\left|\mathcal{T}_{F}\right|^{-\frac{1}{2}} b_{F} \leq\left(\sum_{K \in \mathcal{T}_{h}}\left|\mathcal{F}_{K}\right| a_{K}^{2}\right)^{\frac{1}{2}}\left(\sum_{F \in \mathcal{F}_{h}} b_{F}^{2}\right)^{\frac{1}{2}}$, for all real numbers $\left\{a_{K}\right\}_{K \in \mathcal{T}_{h}},\left\{b_{F}\right\}_{F \in \mathcal{F}_{h}}$, where we used $\sum_{F \in \mathcal{F}_{h}} \sum_{K \in \mathcal{T}_{F}}=\sum_{K \in \mathcal{T}_{h}} \sum_{F \in \mathcal{F}_{K}}$ for the term involving the $a_{K}$ 's. Since $\left|\mathcal{F}_{K}\right|$ is uniformly bounded $\left(\left|\mathcal{F}_{K}\right|=d+1\right.$ for simplicial meshes), applying this bound to the two terms composing the above estimate on $\left|n_{\sharp}\left(v, w_{h}\right)\right|$ leads to (3.21).

Remark 3.6 (Literature). Diffusion-dependent averages have been introduced in Dryja [19] for discontinuous Galerkin methods and have been analyzed, e.g., in Burman and Zunino [10], Dryja et al. [20], Di Pietro et al. [17], Ern et al. [26].

4. Applications. The goal of this section is to perform a unified error analysis for the approximation of the model problem (2.1) with various nonconforming methods: Crouzeix-Raviart finite elements, Nitsche's boundary penalty, interior penalty discontinuous Galerkin, and hybrid high-order methods. We assume in the entire section that Assumption 2.1 holds true. Recall that this implies that, for all $f \in L^{q}(D)$, $q \in\left(\frac{2 d}{2+d}, 2\right]$, the exact solution is in the functional space $V_{\mathrm{S}} \cap H^{1+r}(D)$, with $V_{\mathrm{S}}$ defined in (2.5), $r>0$, and $p>2$ (see (3.2)). Our unified analysis hinges on the dimensionally-consistent seminorm

$$
|v|_{\lambda, p, q}^{2}:=\left\|\lambda^{\frac{1}{2}} \nabla_{h} v\right\|_{L^{2}(D)}^{2}+|v|_{n_{\sharp}}^{2}, \quad \forall v \in V_{\mathrm{S}}+P_{k}^{\mathrm{b}}\left(\mathcal{T}_{h}\right),
$$

with $|\cdot|_{n_{\sharp}}$ defined in (3.18). Since $\lambda$ is piecewise constant, we have

$$
\begin{aligned}
|v|_{\lambda, p, q}^{2}= & \sum_{K \in \mathcal{T}_{h}} \lambda_{K}\left(\left\|\nabla v_{\mid K}\right\|_{L^{2}(K)}^{2}+h_{K}^{2 d\left(\frac{1}{2}-\frac{1}{p}\right)}\left\|\nabla v_{\mid K}\right\|_{L^{p}(K)}^{2}\right. \\
& \left.+h_{K}^{2 d\left(\frac{d+2}{2 d}-\frac{1}{q}\right)}\left\|\Delta v_{\mid K}\right\|_{L^{q}(K)}^{2}\right) .
\end{aligned}
$$

Invoking inverse inequalities shows that there is $c$, uniform w.r.t. $h \in \mathcal{H}$, but depending on $p$ and $q$, s.t.

$$
\left|v_{h}\right|_{\lambda, p, q} \leq c\left\|\lambda^{\frac{1}{2}} \nabla_{h} v_{h}\right\|_{L^{2}(D)}, \quad \forall v_{h} \in P_{k}^{\mathrm{b}}\left(\mathcal{T}_{h}\right) .
$$

4.1. Abstract approximation result. We start by recalling a general approximation result established in [25, Lem. 4.4]. Let $V$ and $W$ be two real Banach spaces. Let $a(\cdot, \cdot)$ be a bounded bilinear form on $V \times W$, and let $\ell(\cdot)$ be a bounded linear form on $W$, i.e., $\ell \in W^{\prime}$. We consider the following abstract model problem:

$$
\left\{\begin{array}{l}
\text { Find } u \in V \text { such that } \\
a(u, w)=\ell(w), \quad \forall w \in W
\end{array}\right.
$$


which we assume to be well-posed in the sense of Hadamard; that is to say, there is a unique solution and this solution depends continuously on the data.

We now formulate a discrete version of the problem (4.4) by using the Galerkin method. We replace the infinite-dimensional spaces $V$ and $W$ by finite-dimensional spaces $V_{h}$ and $W_{h}$ that are members of sequences of spaces $\left(V_{h}\right)_{h \in \mathcal{H}},\left(W_{h}\right)_{h \in \mathcal{H}}$ endowed with some approximation properties as $h \rightarrow 0$. The norms in $V_{h}$ and $W_{h}$ are denoted by $\|\cdot\|_{V_{h}}$ and $\|\cdot\|_{W_{h}}$, respectively. The discrete version of (4.4) is formulated as follows:

$$
\left\{\begin{array}{l}
\text { Find } u_{h} \in V_{h} \text { such that } \\
a_{h}\left(u_{h}, w_{h}\right)=\ell_{h}\left(w_{h}\right), \quad \forall w_{h} \in W_{h},
\end{array}\right.
$$

where $a_{h}(\cdot, \cdot)$ is a bounded bilinear form on $V_{h} \times W_{h}$ and $\ell_{h}(\cdot)$ is a bounded linear form on $W_{h}$; note that $a_{h}(\cdot, \cdot)$ and $\ell_{h}(\cdot)$ possibly differ from $a(\cdot, \cdot)$ and $\ell(\cdot)$, respectively. We henceforth assume that $\operatorname{dim}\left(V_{h}\right)=\operatorname{dim}\left(W_{h}\right)$ and that

$$
\inf _{0 \neq v_{h} \in V_{h}} \sup _{0 \neq w_{h} \in W_{h}} \frac{\left|a_{h}\left(v_{h}, w_{h}\right)\right|}{\left\|v_{h}\right\|_{V_{h}}\left\|w_{h}\right\|_{W_{h}}}=: \alpha_{h}>0, \quad \forall h>0,
$$

so that the discrete problem (4.5) is well-posed.

We formalize the fact that the error analysis requires the solution to (4.4) to be slightly more regular than just being a member of $V$ by introducing a functional space $V_{\mathrm{S}}$ such that $u \in V_{\mathrm{S}} \subsetneq V$. Our setting for the error analysis is therefore as follows:

$$
u \in V_{\mathrm{S}} \subsetneq V, \quad u-u_{h} \in V_{\sharp}:=V_{\mathrm{S}}+V_{h},
$$

with the norm in $V_{\sharp}$ denoted by $\|\cdot\|_{V_{\sharp}}$. Since $V_{h}$ is finite-dimensional, we have

$$
c_{\sharp h}:=\sup _{0 \neq v_{h} \in V_{h}} \frac{\left\|v_{h}\right\|_{V_{\sharp}}}{\left\|v_{h}\right\|_{V_{h}}}<\infty .
$$

Recalling that $u_{h}$ denotes the unique solution to (4.5), we now define the consistency error as the mapping $\delta_{h}: V_{h} \rightarrow W_{h}^{\prime}:=\mathcal{L}\left(W_{h} ; \mathbb{R}\right)$ so that the following holds for all $v_{h} \in V_{h}$ and all $w_{h} \in W_{h}$ :

$$
\left\langle\delta_{h}\left(v_{h}\right), w_{h}\right\rangle_{W_{h}^{\prime}, W_{h}}:=\ell_{h}\left(w_{h}\right)-a_{h}\left(v_{h}, w_{h}\right)=a_{h}\left(u_{h}-v_{h}, w_{h}\right) .
$$

We further assume that

$$
\omega_{\sharp h}:=\sup _{v \in V_{\mathrm{S}}} \sup _{v_{h} \in V_{h} \backslash\{u\}} \frac{\left\|\delta_{h}\left(v_{h}\right)\right\|_{W_{h}^{\prime}}}{\left\|v-v_{h}\right\|_{V_{\sharp}}}<\infty .
$$

The main result we are going to invoke later in the error analysis of nonconforming approximation methods is the following. It can be viewed as a generalization of Strang's second lemma that avoids using the exact solution as an argument of the discrete bilinear form.

LEMma 4.1 (Quasi-optimal error estimate). If $u \in V_{\mathrm{S}}$, then

$$
\left\|u-u_{h}\right\|_{V_{\sharp}} \leq\left(1+c_{\sharp h} \frac{\omega_{\sharp h}}{\alpha_{h}}\right) \inf _{v_{h} \in V_{h}}\left\|u-v_{h}\right\|_{V_{\sharp}} .
$$


Proof. The proof is classical; we sketch it for completeness. For all $v_{h} \in V_{h}$, we have

$$
\begin{aligned}
\left\|u_{h}-v_{h}\right\|_{V_{\sharp}} & \leq c_{\sharp h}\left\|u_{h}-v_{h}\right\|_{V_{h}} \leq \frac{c_{\sharp h}}{\alpha_{h}} \sup _{\substack{0 \neq w_{h} \in W_{h}\\
}} \frac{\left|a_{h}\left(u_{h}-v_{h}, w_{h}\right)\right|}{\left\|w_{h}\right\|_{W_{h}}} \\
& =\frac{c_{\sharp h}}{\alpha_{h}}\left\|\delta_{h}\left(v_{h}\right)\right\|_{W_{h}^{\prime}} \leq \frac{c_{\sharp h} \omega_{\sharp h}}{\alpha_{h}}\left\|u-v_{h}\right\|_{V_{\sharp}} .
\end{aligned}
$$

We conclude by using the triangle inequality and taking the infimum over $v_{h} \in V_{h}$. $\square$

When the constants $c_{\sharp h}$ and $\omega_{\sharp h}$ can be bounded from above uniformly w.r.t. $h \in \mathcal{H}$, we denote by $c_{\sharp}$ and $\omega_{\sharp}$ any constant such that $c_{\sharp} \geq \sup _{h \in \mathcal{H}} c_{\sharp h}$ and $\omega_{\sharp} \geq$ $\sup _{h \in \mathcal{H}} \omega_{\sharp h}$. Notice that Lemma 4.1 does not say anything on how to choose the norms $\|\cdot\|_{V_{h}},\|\cdot\|_{W_{h}},\|\cdot\|_{V_{\sharp}}$ to minimize $\frac{c_{\sharp h} \omega_{\sharp h}}{\alpha_{h}}$.

Example 4.2 (Conforming setting). Assume conformity, $a_{h}=a$, and $\ell_{h}=\ell$. Take $V_{\mathrm{S}}:=V$, so that $V_{\sharp}=V$, and take $\|\cdot\|_{V_{\sharp}}:=\|\cdot\|_{V}$. The consistency error (4.9) is such that

$$
\left\langle\delta_{h}\left(v_{h}\right), w_{h}\right\rangle_{W_{h}^{\prime}, W_{h}}=\ell\left(w_{h}\right)-a\left(v_{h}, w_{h}\right)=a\left(u-v_{h}, w_{h}\right),
$$

where we used that $\ell\left(w_{h}\right)=a\left(u, w_{h}\right)$ (i.e., the Galerkin orthogonality property). Since $a$ is bounded on $V \times W,(4.10)$ holds true with $\omega_{\sharp h}=\|a\|$; moreover, $c_{\sharp h}=1$. Then Lemma 4.1 is just Céa's lemma.

4.2. Crouzeix-Raviart approximation. We consider in this section the approximation of the model problem (2.2) with a homogeneous Dirichlet condition (for simplicity) using the Crouzeix-Raviart finite element space

$$
P_{1,0}^{\mathrm{CR}}\left(\mathcal{T}_{h}\right):=\left\{v_{h} \in P_{1}^{\mathrm{b}}\left(\mathcal{T}_{h}\right) \mid \int_{F} \llbracket v_{h} \rrbracket_{F} \mathrm{~d} s=0, \forall F \in \mathcal{F}_{h}\right\} .
$$

The discrete problem (4.5) is formulated with $V_{h}:=P_{1,0}^{\mathrm{CR}}\left(\mathcal{T}_{h}\right)$ and the following forms:

$$
a_{h}\left(v_{h}, w_{h}\right):=\int_{D} \lambda \nabla_{h} v_{h} \cdot \nabla_{h} w_{h} \mathrm{~d} x, \quad \ell_{h}\left(w_{h}\right):=\int_{D} f w_{h} \mathrm{~d} x .
$$

We equip $V_{h}$ with the norm $\left\|v_{h}\right\|_{V_{h}}:=\left\|\lambda^{\frac{1}{2}} \nabla_{h} v_{h}\right\|_{L^{2}(D)}$. The following result is standard.

Lemma 4.3 (Coercivity, well-posedness). The bilinear form $a_{h}$ is coercive on $V_{h}$ with coercivity constant $\alpha=1$, and the discrete problem (4.5) is well-posed.

Let $V_{\sharp}:=V_{\mathrm{S}}+V_{h}$ be equipped with the norm $\|v\|_{V_{\sharp}}:=|v|_{\lambda, p, q}$ with $|v|_{\lambda, p, q}$ defined in (4.2) (this is indeed a norm on $V_{\sharp}$ since $|v|_{\lambda, p, q}=0$ implies that $v$ is piecewise constant and hence vanishes identically owing to the definition of $V_{h}$ ). Owing to (4.3), there is $c_{\sharp}$, uniform w.r.t. $h \in \mathcal{H}$, but depending on $p$ and $q$, s.t. $\left\|v_{h}\right\|_{V_{\sharp}} \leq c_{\sharp}\left\|v_{h}\right\|_{V_{h}}$ for all $v_{h} \in V_{h}$.

Lemma 4.4 (Consistency/boundedness). Let $\delta_{h}$ be defined in (4.9) with $a_{h}$ and $\ell_{h}$ defined in (4.13). Then, there is $\omega_{\sharp}$ such that $\left\|\delta_{h}\left(v_{h}\right)\right\|_{V_{h}^{\prime}} \leq \omega_{\sharp}\left\|u-v_{h}\right\|_{V_{\sharp}}$ for all $h \in \mathcal{H}$, all $v_{h} \in V_{h}$, all $f \in L^{q}(D)$, and all $\lambda \in \Lambda(\Pi)$, where $u$ is the unique solution to $(2.2)$.

Proof. Let $v_{h}, w_{h} \in V_{h}$. Since $V_{h} \subset P_{k}^{\mathrm{b}}\left(\mathcal{T}_{h}\right)$, the identity (3.16a) implies that

$$
n_{\sharp}\left(v_{h}, w_{h}\right)=\sum_{F \in \mathcal{F}_{h}} \int_{F}\left\{\boldsymbol{\sigma}\left(v_{h}\right)\right\}_{\theta} \cdot \boldsymbol{n}_{F} \llbracket w_{h} \rrbracket \mathrm{d} s=0,
$$


because $\left\{\boldsymbol{\sigma}\left(v_{h}\right)\right\}_{\theta} \cdot \boldsymbol{n}_{F}$ is constant over $F$. Moreover, invoking the identity (3.16b) with $v=u$ and since $f=\nabla \cdot \boldsymbol{\sigma}(u)$, we have

$$
\ell_{h}\left(w_{h}\right)=n_{\sharp}\left(u, w_{h}\right)-\int_{D} \boldsymbol{\sigma}(u) \cdot \nabla_{h} w_{h} \mathrm{~d} x .
$$

Combining the above two identities and letting $\eta:=u-v_{h}$, we obtain

$$
\left\langle\delta_{h}\left(v_{h}\right), w_{h}\right\rangle_{V_{h}^{\prime}, V_{h}}=n_{\sharp}\left(u, w_{h}\right)+\int_{D} \lambda \nabla_{h} \eta \cdot \nabla_{h} w_{h} \mathrm{~d} x=n_{\sharp}\left(\eta, w_{h}\right)+\int_{D} \lambda \nabla_{h} \eta \cdot \nabla_{h} w_{h} \mathrm{~d} x .
$$

The first term on the right-hand side is estimated by invoking the boundedness of $n_{\sharp}$ (Lemma 3.5), the inequality $\lambda_{F} \leq \min _{K \in \mathcal{T}_{F}} \lambda_{K}$ (see (3.20)), and the bound $\sum_{F \in \mathcal{F}_{h}} \lambda_{F} h_{F}^{-1}\left\|\llbracket w_{h} \rrbracket\right\|_{L^{2}(F)}^{2} \leq c\left\|w_{h}\right\|_{V_{h}}^{2}$, which is standard for Crouzeix-Raviart elements. The second term is estimated by using the Cauchy-Schwarz inequality.

TheOREM 4.5 (Error estimate). For all $f \in L^{q}(D)$ and all $\lambda \in \Lambda(\Pi)$, let $u$ denote the solution to (2.2), and let $u_{h} \in V_{h}$ denote the solution to (4.5) with $a_{h}$ and $\ell_{h}$ defined in (4.13). Then, there is $c$ so that the following quasi-optimal error estimate holds true for all $h \in \mathcal{H}$, all $f \in L^{q}(D)$, and all $\lambda \in \Lambda(\Pi)$ :

$$
\left\|u-u_{h}\right\|_{V_{\sharp}} \leq c \inf _{v_{h} \in V_{h}}\left\|u-v_{h}\right\|_{V_{\sharp}} .
$$

Moreover, letting $t:=\min (1, r)$, where $1=k$ is the degree of the Crouzeix-Raviart finite element, we have

$$
\left\|u-u_{h}\right\|_{V_{\sharp}} \leq c\left(\sum_{K \in \mathcal{T}_{h}} \lambda_{K} h_{K}^{2 t}|u|_{H^{1+t}(K)}^{2}+\lambda_{K}^{-1} h_{K}^{2 d\left(\frac{d+2}{2 d}-\frac{1}{q}\right)}\|f\|_{L^{q}(K)}^{2}\right)^{\frac{1}{2}} .
$$

Proof. The error estimate (4.14) follows from Lemma 4.1 combined with stability (Lemma 4.3) and consistency/boundedness (Lemma 4.4). We now bound the infimum in (4.14) by considering $\eta:=u-\mathcal{I}_{h}^{\mathrm{CR}}(u)$, where $\mathcal{I}_{h}^{\mathrm{CR}}$ is the Crouzeix-Raviart interpolation operator using averages over the faces as degrees of freedom. It is a standard approximation result that there is $c$, uniform w.r.t. $u \in H^{1+t}(K), t \geq 0$, and $h \in \mathcal{H}$, s.t. $\left\|\nabla \eta_{\mid K}\right\|_{L^{2}(K)} \leq c h_{K}^{t}|u|_{H^{1+t}(K)}$ for all $K \in \mathcal{T}_{h}$. Moreover, invoking the embedding $\boldsymbol{H}^{t}(\widehat{K}) \hookrightarrow \boldsymbol{L}^{p}(\widehat{K})$ and classical results on the transformation of Sobolev norms by the geometric mapping, we obtain the bound

$$
h_{K}^{d\left(\frac{1}{2}-\frac{1}{p}\right)}\left\|\nabla \eta_{\mid K}\right\|_{\boldsymbol{L}^{p}(K)} \leq c\left(\left\|\nabla \eta_{\mid K}\right\|_{\boldsymbol{L}^{2}(K)}+h_{K}^{t}\left|\nabla \eta_{\mid K}\right|_{\boldsymbol{H}^{t}(K)}\right) .
$$

Observing that $\left|\nabla \eta_{\mid K}\right|_{H^{t}(K)}=|u|_{H^{1+t}(K)}$ since $\mathcal{I}_{h}^{\mathrm{CR}}(u)$ is affine on $K$ and using again the approximation properties of $\mathcal{I}_{h}^{\mathrm{CR}}$, we infer that $h_{K}^{d\left(\frac{1}{2}-\frac{1}{p}\right)}\left\|\nabla \eta_{\mid K}\right\|_{\boldsymbol{L}^{p}(K)} \leq$ $c h_{K}^{t}|u|_{H^{1+t}(K)}$. Finally, we have $\Delta \eta_{\mid K}=\lambda_{K}^{-1} f$ in $K$.

Remark 4.6 (Convergence). The rightmost term in (4.15) converges as $O(h)$ when $q=2$. Moreover, convergence is lost when $q \leq \frac{2 d}{d+2}$, which is somewhat natural since in this case the linear form $w \mapsto \int_{D} f w \mathrm{~d} x$ is no longer bounded on $H^{1}(D)$.

Remark 4.7 (Weights). Although the weights introduced in (3.19) are not explicitly used in the Crouzeix-Raviart discretization, they play a role in the error analysis. More precisely, we used the boundedness of the bilinear form $n_{\sharp}$ together with $\lambda_{F} \leq \min _{K \in \mathcal{T}_{F}} \lambda_{K}$ in the proof of Lemma 4.4. The present approach is somewhat more general than that in Li and Mao [31] since it delivers error estimates that are robust with respect to the diffusivity contrast. The trimming operator invoked in [31, Eq. (5)-(7)] cannot account for the diffusivity contrast. 
4.3. Nitsche's boundary penalty method. We consider in this section the approximation of the model problem (2.1) by means of Nitsche's boundary penalty method. Now we set

$$
V_{h}:=P_{k}^{\mathrm{g}}\left(\mathcal{T}_{h}\right):=\left\{v_{h} \in P_{k}^{\mathrm{b}}\left(\mathcal{T}_{h}\right) \mid \llbracket v_{h} \rrbracket_{F}=0, \forall F \in \mathcal{F}_{h}^{\circ}\right\}, \quad k \geq 1,
$$

i.e., $V_{h}$ is $H^{1}$-conforming. The discrete problem (4.5) is formulated with $V_{h}:=P_{k}^{\mathrm{g}}\left(\mathcal{T}_{h}\right)$ and the following forms:

$$
\begin{aligned}
a_{h}\left(v_{h}, w_{h}\right) & :=a\left(v_{h}, w_{h}\right)+\sum_{F \in \mathcal{F}_{h}^{\partial}} \int_{F}\left(\boldsymbol{\sigma}\left(v_{h}\right) \cdot \boldsymbol{n}+\varpi_{0} \frac{\lambda_{K_{l}}}{h_{F}} v_{h}\right) w_{h} \mathrm{~d} s, \\
\ell_{h}\left(w_{h}\right) & :=\ell\left(w_{h}\right)+\sum_{F \in \mathcal{F}_{h}^{\partial}} \varpi_{0} \frac{\lambda_{K_{l}}}{h_{F}} \int_{F} g w_{h} \mathrm{~d} s,
\end{aligned}
$$

where the exact forms $a$ and $\ell$ are defined in (2.3), $K_{l}$ is the unique mesh cell s.t. $F=\partial K_{l} \cap \partial D$, and the user-specified penalty parameter $\varpi_{0}$ is yet to be chosen large enough. It is possible to add a symmetrizing term to the discrete bilinear form $a_{h}$.

We equip $V_{h}$ with the norm $\left\|v_{h}\right\|_{V_{h}}^{2}:=\left\|\lambda^{\frac{1}{2}} \nabla v_{h}\right\|_{L^{2}(D)}^{2}+\left|v_{h}\right|_{\partial}^{2}$ with $\left|v_{h}\right|_{\partial}^{2}:=$ $\sum_{F \in \mathcal{F}_{h}^{2}} \frac{\lambda_{K_{l}}}{h_{F}}\left\|v_{h}\right\|_{L^{2}(F)}^{2}$. Owing to the shape-regularity of the mesh sequence, there is $c_{I}$, uniform w.r.t. $h \in \mathcal{H}$, s.t.

$$
\left\|v_{h}\right\|_{L^{2}(F)} \leq c_{I} h_{F}^{-\frac{1}{2}}\left\|v_{h}\right\|_{L^{2}\left(K_{l}\right)},
$$

for all $v_{h} \in V_{h}$ and all $F \in \mathcal{F}_{h}^{\partial}$. Let $n_{\partial}$ denote the maximum number of boundary faces that a mesh cell can have ( $n_{\partial} \leq d$ for simplicial meshes). The proof of the following result uses standard arguments.

Lemma 4.8 (Coercivity, well-posedness). Assume that the penalty parameter satisfies $\varpi_{0}>\frac{1}{4} n_{\partial} c_{I}^{2}$. Then, $a_{h}$ is coercive on $V_{h}$ with constant $\alpha:=\frac{\varpi_{0}-\frac{1}{4} n_{\partial} c_{I}^{2}}{1+\varpi_{0}}>0$, and the discrete problem (4.5) is well-posed.

Let $V_{\sharp}:=V_{\mathrm{S}}+V_{h}$. We equip the space $V_{\sharp}$ with the norm $\|v\|_{V_{\sharp}}^{2}:=|v|_{\lambda, p, q}^{2}+|v|_{\partial}^{2}$ where the definition of the seminorm $|\cdot|_{\lambda, p, q}$ is slightly changed as follows:

$$
\begin{aligned}
|v|_{\lambda, p, q}^{2}:= & \sum_{K \in \mathcal{T}_{h}} \lambda_{K}\left\|\nabla v_{\mid K}\right\|_{L^{2}(K)}^{2} \\
& +\sum_{K \in \overline{\mathcal{T}}_{h}^{\partial}} \lambda_{K}\left(h_{K}^{2 d\left(\frac{1}{2}-\frac{1}{p}\right)}\left\|\nabla v_{\mid K}\right\|_{\boldsymbol{L}^{p}(K)}^{2}+h_{K}^{2 d\left(\frac{d+2}{2 d}-\frac{1}{q}\right)}\left\|\Delta v_{\mid K}\right\|_{L^{q}(K)}^{2}\right),
\end{aligned}
$$

where $\overline{\mathcal{T}}_{h}^{\partial}$ is the collection of the mesh cells having at least one boundary face, and $|v|_{\partial}^{2}=\sum_{F \in \mathcal{F}_{h}^{\partial}} \frac{\lambda_{K_{l}}}{h_{F}}\|v\|_{L^{2}(F)}^{2}$. (The second summation in (4.20) is restricted to $K \in \overline{\mathcal{T}}_{h}^{\partial}$ since only those cells are concerned by the bound on the consistency error for Nitsche's boundary penalty method.) Owing to (4.3), there is $c_{\sharp}$, uniform w.r.t. $h \in \mathcal{H}$, but depending on $p$ and $q$, s.t. $\left\|v_{h}\right\|_{V_{\sharp}} \leq c_{\sharp}\left\|v_{h}\right\|_{V_{h}}$ for all $v_{h} \in V_{h}$.

Lemma 4.9 (Consistency/boundedness). Let $\delta_{h}$ be defined in (4.9) with $a_{h}$ and $\ell_{h}$ defined in (4.18). Then, there is $\omega_{\sharp}$ such that $\left\|\delta_{h}\left(v_{h}\right)\right\|_{V_{h}^{\prime}} \leq \omega_{\sharp}\left\|u-v_{h}\right\|_{V_{\sharp}}$ for all $h \in \mathcal{H}$, all $v_{h} \in V_{h}$, all $f \in L^{q}(D)$, and all $\lambda \in \Lambda(\Pi)$, where $u$ is the unique solution to $(2.2)$. 
Proof. Let $v_{h}, w_{h} \in V_{h}$. Using the identity (3.16a) for $n_{\sharp}, \llbracket w_{h} \rrbracket_{F}=0$ for all $F \in \mathcal{F}_{h}^{\circ}$ (since $V_{h}$ is $H^{1}$-conforming), and the definition of the weights at the boundary faces, we infer that $n_{\sharp}\left(v_{h}, w_{h}\right)=\sum_{F \in \mathcal{F}_{h}^{\partial}} \int_{F} \boldsymbol{\sigma}\left(v_{h}\right) \cdot \boldsymbol{n} w_{h} \mathrm{~d} s$. Hence, $a_{h}\left(v_{h}, w_{h}\right)=$ $a\left(v_{h}, w_{h}\right)+n_{\sharp}\left(v_{h}, w_{h}\right)+\sum_{F \in \mathcal{F}_{h}^{\partial}} \varpi_{0} \frac{\lambda_{K_{l}}}{h_{F}} \int_{F} v_{h} w_{h} \mathrm{~d} s$. Therefore, invoking the identity (3.16b) for the exact solution $u$ and observing that $f=\nabla \cdot \boldsymbol{\sigma}(u)$, we infer the important identity $\int_{D} f w_{h} \mathrm{~d} x=a\left(u, w_{h}\right)+n_{\sharp}\left(u, w_{h}\right)$. Then, recalling that $\gamma^{\mathrm{g}}(u)=g$, and letting $\eta:=u-v_{h}$, we obtain

$$
\left\langle\delta_{h}\left(v_{h}\right), w_{h}\right\rangle_{V_{h}^{\prime}, V_{h}}=n_{\sharp}\left(\eta, w_{h}\right)+a\left(\eta, w_{h}\right)+\sum_{F \in \mathcal{F}_{h}^{\partial}} \varpi_{0} \frac{\lambda_{K_{l}}}{h_{F}} \int_{F} \eta w_{h} \mathrm{~d} s .
$$

We conclude by using the boundedness of $n_{\sharp}$ from Lemma 3.5 and the Cauchy-Schwarz inequality.

Theorem 4.10 (Error estimate). For all $f \in L^{q}(D)$ and all $\lambda \in \Lambda(\Pi)$, let $u$ denote the solution to (2.2), and let $u_{h} \in V_{h}$ denote the solution to (4.5) with $a_{h}$ and $\ell_{h}$ defined in (4.18). Then, there is $c$ so that the following quasi-optimal error estimate holds true for all $h \in \mathcal{H}$, all $f \in L^{q}(D)$, and all $\lambda \in \Lambda(\Pi)$ :

$$
\left\|u-u_{h}\right\|_{V_{\sharp}} \leq c \inf _{v_{h} \in V_{h}}\left\|u-v_{h}\right\|_{V_{\sharp}} .
$$

Moreover, letting $t:=\min (r, k), \chi_{t}:=1$ if $t \leq 1$ and $\chi_{t}:=0$ if $t>1$, we have

$$
\left\|u-u_{h}\right\|_{V_{\sharp}} \leq c\left(\sum_{K \in \mathcal{T}_{h}} \lambda_{K} h_{K}^{2 t}|u|_{H^{1+t}\left(\check{\mathcal{T}}_{K}\right)}^{2}+\frac{\chi_{t}}{\lambda_{K}} h_{K}^{2 d\left(\frac{d+2}{2 d}-\frac{1}{q}\right)}\|f\|_{L^{q}(K)}^{2}\right)^{\frac{1}{2}},
$$

where $\check{\mathcal{T}}_{K}$ is the collection of the mesh cells having at least a common vertex with $K$. The broken Sobolev norm $|\cdot|_{H^{1+t}\left(\check{\mathcal{T}}_{K}\right)}$ can be replaced by $|\cdot|_{H^{1+t}(K)}$ if $1+t>\frac{d}{2}$.

Proof. The error estimate (4.21) follows from Lemma 4.1 combined with stability (Lemma 4.8) and consistency/boundedness (Lemma 4.9). We now bound the infimum in (4.21) by using $\eta:=u-\mathcal{I}_{h}^{\text {g,av }}(u)$, where $\mathcal{I}_{h}^{\text {g,av }}$ is the quasi-interpolation operator introduced in $[23, \S 5]$. We take the polynomial degree of $\mathcal{I}_{h}^{\mathrm{g}, \text { av }}$ to be $\ell:=\lceil t\rceil$, where $\lceil t\rceil$ denotes the smallest integer $n \in \mathbb{N}$ s.t. $n \geq t$. Notice that $\ell \geq 1$ because $r>0$ and $k \geq 1$, and $\ell \leq k$ because $t \leq k$; hence, $\mathcal{I}_{h}^{\text {g,av }}(u) \in V_{h}$. We need to bound all the terms composing the norm $\|\eta\|_{V_{\sharp}}$. Owing to [23, Thm. 5.2] with $m=1$, we have $\|\nabla \eta\|_{L^{2}(K)} \leq c h_{K}^{t}|u|_{H^{1+t}\left(\check{\mathcal{T}}_{K}\right)}$ for all $K \in \mathcal{T}_{h}$. Moreover, we have $h_{F}^{-\frac{1}{2}}\|\eta\|_{L^{2}(F)} \leq c h_{K_{l}}^{t}|u|_{H^{1+t}\left(\check{\mathcal{T}}_{K_{l}}\right)}$ for all $F \in \mathcal{F}_{h}^{\partial}$. It remains to estimate $h_{K}^{d\left(\frac{1}{2}-\frac{1}{p}\right)}\left\|\nabla \eta_{\mid K}\right\|_{\boldsymbol{L}^{p}(K)}$ and $h_{K}^{d\left(\frac{d+2}{2 d}-\frac{1}{q}\right)}\left\|\Delta \eta_{\mid K}\right\|_{L^{q}(K)}$ for all $K \in \overline{\mathcal{T}}_{h}^{\partial}$. Using (4.16), the above bound on $\|\nabla \eta\|_{L^{2}(K)}$, and $|\nabla \eta|_{\boldsymbol{H}^{t}(K)}=|\nabla u|_{\boldsymbol{H}^{t}(K)}=|u|_{H^{1+t}(K)}$ since $\ell<1+t$, we infer that $h_{K}^{d\left(\frac{1}{2}-\frac{1}{p}\right)}\|\nabla \eta\|_{L^{p}(K)} \leq c h_{K}^{t}|u|_{H^{1+t}\left(\check{\mathcal{T}}_{K}\right)}$. Moreover, if $t \leq 1$, we have $\ell=1$ so that $\left\|\Delta \eta_{\mid K}\right\|_{L^{q}(K)}=\|\Delta u\|_{L^{q}(K)}=\lambda_{K}^{-1}\|f\|_{L^{q}(K)}$. Instead, if $t>1$, we infer that $r>1$ so that we can set $q=2$ (recall that $f_{\mid D_{i}}=\lambda_{\mid D_{i}}(\Delta u)_{D_{i}}$ for all $i \in\{1: M\}$, and $u \in H^{2}(D)$ if $r \geq 1$ ), and we estimate $\left\|\Delta \eta_{\mid K}\right\|_{L^{2}(K)}$ using [23, Thm. 5.2] with $m=2$. Finally, if $1+t>\frac{d}{2}$, we can use the canonical Lagrange interpolation operator $\mathcal{I}_{h}^{\mathrm{g}}$ instead of $\mathcal{I}_{h}^{\text {g,av }}$, and this allows us to replace $|\cdot|_{H^{1+t}\left(\check{\mathcal{T}}_{K}\right)}$ by $|\cdot|_{H^{1+t}(K)}$ in (4.22).

4.4. Discontinuous Galerkin. We consider in this section the approximation of the model problem (2.1) by means of the symmetric interior penalty discontinuous 
Galerkin method. The discrete problem (4.5) is formulated with $V_{h}:=P_{k}^{\mathrm{b}}\left(\mathcal{T}_{h}\right), k \geq 1$, the bilinear forms

$$
\begin{aligned}
a_{h}\left(v_{h}, w_{h}\right):= & \int_{D} \lambda \nabla_{h} v_{h} \cdot \nabla_{h} w_{h} \mathrm{~d} x+\sum_{F \in \mathcal{F}_{h}} \int_{F}\left\{\boldsymbol{\sigma}\left(v_{h}\right)\right\}_{\theta} \cdot \boldsymbol{n}_{F} \llbracket w_{h} \rrbracket \mathrm{d} s \\
& +\sum_{F \in \mathcal{F}_{h}} \int_{F} \llbracket v_{h} \rrbracket\left\{\boldsymbol{\sigma}\left(w_{h}\right)\right\}_{\theta} \cdot \boldsymbol{n}_{F} \mathrm{~d} s+\sum_{F \in \mathcal{F}_{h}} \varpi_{0} \frac{\lambda_{F}}{h_{F}} \int_{F} \llbracket v_{h} \rrbracket \llbracket w_{h} \rrbracket \mathrm{d} s \\
\ell_{h}\left(w_{h}\right):= & \ell\left(w_{h}\right)+\sum_{F \in \mathcal{F}_{h}^{\partial}} \varpi_{0} \frac{\lambda_{K_{l}}}{h_{F}} \int_{F} g w_{h} \mathrm{~d} s
\end{aligned}
$$

where $\ell$ is defined in (2.3), $\lambda_{F}$ in (3.20), and the user-specified penalty parameter $\varpi_{0}$ is yet to be chosen large enough. We equip $V_{h}$ with the norm $\left\|v_{h}\right\|_{V_{h}}^{2}:=$ $\left\|\lambda^{\frac{1}{2}} \nabla_{h} v_{h}\right\|_{L^{2}(D)}^{2}+\left|v_{h}\right|_{J}^{2}$ with $\left|v_{h}\right|_{J}^{2}:=\sum_{F \in \mathcal{F}_{h}} \frac{\lambda_{F}}{h_{F}}\left\|\llbracket v_{h} \rrbracket\right\|_{L^{2}(F)}^{2}$. Recall the discrete trace inequality (4.19) and let $n_{\partial}$ denote the maximum number of faces that a mesh cell can have ( $n_{\partial} \leq d+1$ for simplicial meshes). The proof of the following result uses standard arguments.

Lemma 4.11 (Coercivity, well-posedness). Assume that the penalty parameter satisfies $\varpi_{0}>n_{\partial} c_{I}^{2}$. Then, $a_{h}$ is coercive on $V_{h}$ with constant $\alpha:=\frac{\varpi_{0}-n_{\partial} c_{I}^{2}}{1+\varpi_{0}}>0$, and the discrete problem (4.5) is well-posed.

Let $V_{\sharp}:=V_{\mathrm{S}}+V_{h}$. We equip the space $V_{\sharp}$ with the norm $\|v\|_{V_{\sharp}}^{2}:=|v|_{\lambda, p, q}^{2}+|v|_{J}^{2}$ with $|v|_{\lambda, p, q}$ defined in (4.2) and $|v|_{\mathrm{J}}^{2}:=\sum_{F \in \mathcal{F}_{h}} \frac{\lambda_{F}}{h_{F}}\|\llbracket v \rrbracket\|_{L^{2}(F)}^{2}$. Owing to (4.3), there is $c_{\sharp}$, uniform w.r.t. $h \in \mathcal{H}$, but depending on $p$ and $q$, s.t. $\left\|v_{h}\right\|_{V_{\sharp}} \leq c_{\sharp}\left\|v_{h}\right\|_{V_{h}}$ for all $v_{h} \in V_{h}$.

Lemma 4.12 (Consistency/boundedness). Let $\delta_{h}$ be defined in (4.9) with $a_{h}$ and $\ell_{h}$ defined in (4.23). Then, there is $\omega_{\sharp}$ such that $\left\|\delta_{h}\left(v_{h}\right)\right\|_{V_{h}^{\prime}} \leq \omega_{\sharp}\left\|u-v_{h}\right\|_{V_{\sharp}}$ for all $h \in \mathcal{H}$, all $v_{h} \in V_{h}$, all $f \in L^{q}(D)$, and all $\lambda \in \Lambda(\Pi)$, where $u$ is the unique solution to $(2.2)$.

Proof. Let $v_{h}, w_{h} \in V_{h}$. Owing to (3.16b) and since $f=\nabla \cdot \boldsymbol{\sigma}(u)$, we infer that $\int_{D} f w_{h} \mathrm{~d} x=\sum_{K \in \mathcal{T}_{h}} a_{K}\left(u, w_{h}\right)+n_{\sharp}\left(u, w_{h}\right)$ with $a_{K}\left(u, w_{h}\right):=-\left(\boldsymbol{\sigma}(u), \nabla_{h} w_{h}\right)_{\boldsymbol{L}^{2}(K)}$. Using the identity (3.16a), we obtain

$$
\begin{aligned}
\ell_{h}\left(w_{h}\right)= & n_{\sharp}\left(u, w_{h}\right)-\int_{D} \boldsymbol{\sigma}(u) \cdot \nabla_{h} w_{h} \mathrm{~d} x+\sum_{F \in \mathcal{F}_{h}^{\partial}} \varpi_{0} \frac{\lambda_{F}}{h_{F}} \int_{F} g w_{h} \mathrm{~d} s, \\
a_{h}\left(v_{h}, w_{h}\right)= & \int_{D}-\boldsymbol{\sigma}\left(v_{h}\right) \cdot \nabla_{h} w_{h} \mathrm{~d} x+n_{\sharp}\left(v_{h}, w_{h}\right) \\
& -\sum_{F \in \mathcal{F}_{h}} \int_{F} \llbracket v_{h} \rrbracket\left\{\boldsymbol{\sigma}\left(w_{h}\right)\right\}_{\theta} \cdot \boldsymbol{n}_{F} \mathrm{~d} s+\sum_{F \in \mathcal{F}_{h}} \varpi_{0} \frac{\lambda_{F}}{h_{F}} \int_{F} \llbracket v_{h} \rrbracket \llbracket w_{h} \rrbracket \mathrm{d} s .
\end{aligned}
$$

Then, setting $\eta:=u-v_{h}$ and using that $\llbracket u \rrbracket_{F}=0$ for all $F \in \mathcal{F}_{h}^{\circ}$ and $\llbracket u \rrbracket_{F}=g$ for all $F \in \mathcal{F}_{h}^{\partial}$, we obtain the following representation of the consistency linear form $\delta_{h}\left(v_{h}\right)$ :

$$
\begin{aligned}
\left\langle\delta_{h}\left(v_{h}\right), w_{h}\right\rangle_{V_{h}^{\prime}, V_{h}}= & n_{\sharp}\left(\eta, w_{h}\right)+\int_{D} \lambda \nabla \eta \cdot \nabla_{h} w_{h} \mathrm{~d} x \\
& -\sum_{F \in \mathcal{F}_{h}} \int_{F} \llbracket \eta \rrbracket\left\{\boldsymbol{\sigma}\left(w_{h}\right)\right\}_{\theta} \cdot \boldsymbol{n}_{F} \mathrm{~d} s+\sum_{F \in \mathcal{F}_{h}} \varpi_{0} \frac{\lambda_{F}}{h_{F}} \int_{F} \llbracket \eta \rrbracket \llbracket w_{h} \rrbracket \mathrm{d} s .
\end{aligned}
$$


Bounding the second, third and fourth terms uses standard arguments (see, e.g., [15]), whereas we invoke the boundedness estimate on $n_{\sharp}$ from Lemma 3.5 for the first term. $\square$

TheOREM 4.13 (Error estimate). For all $f \in L^{q}(D)$ and all $\lambda \in \Lambda(\Pi)$, let $u$ denote the solution to (2.2), and let $u_{h} \in V_{h}$ denote the solution to (4.5) with $a_{h}$ and $\ell_{h}$ defined in (4.23). Then, there is $c$ so that the following quasi-optimal error estimate holds true for all $h \in \mathcal{H}$, all $f \in L^{q}(D)$, and all $\lambda \in \Lambda(\Pi)$ :

$$
\left\|u-u_{h}\right\|_{V_{\sharp}} \leq c \inf _{v_{h} \in V_{h}}\left\|u-v_{h}\right\|_{V_{\sharp}} .
$$

Moreover, letting $t:=\min (r, k), \chi_{t}:=1$ if $t \leq 1$ and $\chi_{t}:=0$ if $t>1$, we have

$$
\left\|u-u_{h}\right\|_{V_{\sharp}} \leq c\left(\sum_{K \in \mathcal{T}_{h}} \lambda_{K} h_{K}^{2 t}|u|_{H^{1+t}(K)}^{2}+\frac{\chi_{t}}{\lambda_{K}} h_{K}^{2 d\left(\frac{d+2}{2 d}-\frac{1}{q}\right)}\|f\|_{L^{q}(K)}^{2}\right)^{\frac{1}{2}} .
$$

Proof. We proceed as in the proof of Theorem 4.10, where we now use the $L^{1}$ stable interpolation operator $\mathcal{I}_{h}^{\sharp}: L^{1}(D) \rightarrow P_{k}^{\mathrm{b}}\left(\mathcal{T}_{h}\right)$ from $[23, \S 3]$ to estimate the best-approximation error.

4.5. Hybrid high-order methods. We consider in this section the approximation of the model problem (2.1) with a homogeneous Dirichlet condition (for simplicity) by means of the hybrid high-order (HHO) method introduced in [16, 18]. We consider the discrete product space $\hat{V}_{h, 0}^{k}:=V_{\mathcal{T}_{h}}^{k} \times V_{\mathcal{F}_{h}}^{k}$ with $k \geq 0$, where

$$
\begin{aligned}
V_{\mathcal{T}_{h}}^{k} & :=\left\{v_{\mathcal{T}_{h}} \in L^{2}(D) \mid v_{K}:=v_{\mathcal{T}_{h} \mid K} \in V_{K}^{k}, \forall K \in \mathcal{T}_{h}\right\}, \\
V_{\mathcal{F}_{h}}^{k} & :=\left\{v_{\mathcal{F}_{h}} \in L^{2}\left(\mathcal{F}_{h}\right) \mid v_{\partial K}:=v_{\mathcal{F}_{h} \mid \partial K} \in V_{\partial K}^{k}, \forall K \in \mathcal{T}_{h} ; v_{\mathcal{F}_{h} \mid \mathcal{F}_{h}^{\partial}}=0\right\},
\end{aligned}
$$

with $V_{K}^{k}:=\mathbb{P}_{k, d}$ and $V_{\partial K}^{k}:=\left\{\theta \in L^{2}(\partial K) \mid \theta \circ \boldsymbol{T}_{K \mid \boldsymbol{T}_{K}^{-1}(F)} \in \mathbb{P}_{k, d-1}, \forall F \in \mathcal{F}_{K}\right\}$. Thus, for any pair $\hat{v}_{h}:=\left(v_{\mathcal{T}_{h}}, v_{\mathcal{F}_{h}}\right) \in \hat{V}_{h, 0}^{k}, v_{\mathcal{T}_{h}}$ a collection of cell polynomials of degree at most $k$, and $v_{\mathcal{F}_{h}}$ is a collection of face polynomials of degree at most $k$ which are single-valued at the mesh interfaces and vanish at the boundary faces (so as to enforce strongly the homogeneous Dirichlet condition). We use the notation $\hat{v}_{K}:=\left(v_{K}, v_{\partial K}\right) \in \hat{V}_{K}^{k}:=V_{K}^{k} \times V_{\partial K}^{k}$ for all $K \in \mathcal{T}_{h}$. We equip the local space $\hat{V}_{K}^{k}$ with the $H^{1}$-like seminorm

$$
\left|\hat{v}_{K}\right|_{\hat{V}_{K}^{k}}^{2}:=\left\|\nabla v_{K}\right\|_{L^{2}(K)}^{2}+h_{K}^{-1}\left\|v_{K}-v_{\partial K}\right\|_{L^{2}(\partial K)}^{2}, \quad \forall \hat{v}_{K}=\left(v_{K}, v_{\partial K}\right) \in \hat{V}_{K}^{k},
$$

and the global space $\hat{V}_{h, 0}^{k}$ with the norm

$$
\left\|\hat{v}_{h}\right\|_{\hat{V}_{h, 0}^{k}}^{2}:=\sum_{K \in \mathcal{T}_{h}} \lambda_{K}\left|\hat{v}_{K}\right|_{\hat{V}_{K}^{k}}^{2}
$$

We introduce locally in each mesh cell $K \in \mathcal{T}_{h}$ a reconstruction operator and a stabilization operator. The reconstruction operator $\mathrm{R}_{K}^{k+1}: \hat{V}_{K}^{k} \rightarrow \mathbb{P}_{k+1, d}$ is defined such that, for any pair $\hat{v}_{K}=\left(v_{K}, v_{\partial K}\right) \in \hat{V}_{K}^{k}$, the polynomial function $\mathrm{R}_{K}^{k+1}\left(\hat{v}_{K}\right) \in$ $\mathbb{P}_{k+1, d}$ solves

$$
\left(\nabla \mathrm{R}_{K}^{k+1}\left(\hat{v}_{K}\right), \nabla q\right)_{\boldsymbol{L}^{2}(K)}:=-\left(v_{K}, \Delta q\right)_{L^{2}(K)}+\left(v_{\partial K}, \boldsymbol{n}_{K} \cdot \nabla q\right)_{L^{2}(\partial K)},
$$

for all $q \in \mathbb{P}_{k+1, d}$, with the mean-value condition $\int_{K}\left(\mathrm{R}_{K}^{k+1}\left(\hat{v}_{K}\right)-v_{K}\right) \mathrm{d} x=0$. This local Neumann problem makes sense since the right-hand side of (4.29) vanishes when 
the test function $q$ is constant. The stabilization operator $\mathrm{S}_{\partial K}^{k}: \hat{V}_{K}^{k} \rightarrow V_{\partial K}^{k}$ is defined s.t. for any pair $\hat{v}_{K}=\left(v_{K}, v_{\partial K}\right) \in \hat{V}_{K}^{k}$,

$$
\mathrm{S}_{\partial K}^{k}\left(\hat{v}_{K}\right):=\Pi_{\partial K}^{k}\left(v_{K \mid \partial K}-v_{\partial K}+\left(\left(I-\Pi_{K}^{k}\right) \mathrm{R}_{K}^{k+1}\left(\hat{v}_{K}\right)\right)_{\mid \partial K}\right),
$$

where $I$ is the identity, $\Pi_{\partial K}^{k}: L^{2}(\partial K) \rightarrow V_{\partial K}^{k}$ is the $L^{2}$-orthogonal projection onto $V_{\partial K}^{k}$ and $\Pi_{K}^{k}: L^{2}(K) \rightarrow V_{K}^{k}$ is the $L^{2}$-orthogonal projection onto $V_{K}^{k}$. Elementary algebra shows that the stabilization operator can be rewritten as

$$
\mathrm{S}_{\partial K}^{k}\left(\hat{v}_{K}\right)=\Pi_{\partial K}^{k}\left(\delta_{\partial K}-\left(\left(I-\Pi_{K}^{k}\right) \mathrm{R}_{K}^{k+1}\left(0, \delta_{\partial K}\right)\right)_{\mid \partial K}\right),
$$

with $\delta_{\partial K}:=v_{K \mid \partial K}-v_{\partial K}$ is a measure of the discrepancy between the trace of the cell unknown and the face unknown.

We now introduce the local bilinear form $\hat{a}_{K}$ on $\hat{V}_{K}^{k} \times \hat{V}_{K}^{k}$ s.t.

$$
\begin{aligned}
\hat{a}_{K}\left(\hat{v}_{K}, \hat{w}_{K}\right):=\left(\nabla \mathrm{R}_{K}^{k+1}\left(\hat{v}_{K}\right), \nabla \mathrm{R}_{K}^{k+1}\left(\hat{w}_{K}\right)\right)_{L^{2}(K)} \\
+h_{K}^{-1}\left(\mathrm{~S}_{\partial K}^{k}\left(\hat{v}_{K}\right), \mathrm{S}_{\partial K}^{k}\left(\hat{w}_{K}\right)\right)_{L^{2}(\partial K)} .
\end{aligned}
$$

Then we set

$$
\hat{a}_{h}\left(\hat{v}_{h}, \hat{w}_{h}\right):=\sum_{K \in \mathcal{T}_{h}} \lambda_{K} \hat{a}_{K}\left(\hat{v}_{K}, \hat{w}_{K}\right), \quad \hat{\ell}_{h}\left(\hat{w}_{h}\right):=\sum_{K \in \mathcal{T}_{h}} \int_{K} f w_{K} \mathrm{~d} x .
$$

The discrete problem is formulated as follows: Find $\hat{u}_{h} \in \hat{V}_{h, 0}^{k}$ s.t.

$$
\hat{a}_{h}\left(\hat{u}_{h}, \hat{w}_{h}\right)=\hat{\ell}_{h}\left(\hat{w}_{h}\right), \quad \forall \hat{w}_{h} \in \hat{V}_{h, 0}^{k}
$$

Notice that HHO methods are somewhat simpler than dG methods when it comes to solving problems with contrasted coefficients. For HHO methods, one assembles cellwise the local bilinear forms $\hat{a}_{K}$ weighted by the local diffusion coefficient $\lambda_{K}$, whereas, for $\mathrm{dG}$ methods one has to invoke interface-based values of the diffusion coefficient to construct the penalty term.

The following result is proved in $[16,18]$.

Lemma 4.14 (Stability, boundedness, well-posedness). There exist two positive numbers, $0<\alpha \leq \omega$, so that the following holds:

$$
\alpha\left|\hat{v}_{K}\right|_{\hat{V}_{K}^{k}}^{2} \leq\left\|\nabla \mathrm{R}_{K}^{k+1}\left(\hat{v}_{K}\right)\right\|_{L^{2}(K)}^{2}+h_{K}^{-1}\left\|\mathrm{~S}_{\partial K}^{k}\left(\hat{v}_{K}\right)\right\|_{L^{2}(\partial K)}^{2}=\hat{a}_{K}\left(\hat{v}_{K}, \hat{v}_{K}\right) \leq \omega\left|\hat{v}_{K}\right|_{\hat{V}_{K}^{k}}^{2},
$$

for all $\hat{v}_{K} \in \hat{V}_{K}$, all $K \in \mathcal{T}_{h}$, and all $h \in \mathcal{H}$. Moreover, the discrete problem (4.34) is well-posed.

The two key tools in the error analysis of HHO methods are a local reduction operator and the local elliptic projection. For all $K \in \mathcal{T}_{h}$, the local reduction operator $\hat{\mathcal{I}}_{K}^{k}: H^{1}(K) \rightarrow \hat{V}_{K}^{k}$ is defined by $\hat{\mathcal{I}}_{K}^{k}(v):=\left(\Pi_{K}^{k}(v), \Pi_{\partial K}^{k}\left(\gamma_{\partial K}^{\mathrm{g}}(v)\right)\right) \in \hat{V}_{K}^{k}$, for all $v \in H^{1}(K)$. The local elliptic projection $\mathcal{E}_{K}^{k+1}: H^{1}(K) \rightarrow \mathbb{P}_{k+1, d}$ is s.t. $\left(\nabla\left(\mathcal{E}_{K}^{k+1}(v)-\right.\right.$ $v), \nabla q)_{L^{2}(K)}=0$, for all $q \in \mathbb{P}_{k+1, d}$, and $\left(\mathcal{E}_{K}^{k+1}(v)-v, 1\right)_{L^{2}(K)}=0$. The following result is established in $[16,18]$.

Lemma 4.15 (Polynomial invariance). The following holds true:

$$
\begin{aligned}
\mathrm{R}_{K}^{k+1} \circ \hat{\mathcal{I}}_{K}^{k} & =\mathcal{E}_{K}^{k+1}, \\
\mathrm{~S}_{\partial K}^{k} \circ \hat{\mathcal{I}}_{K}^{k} & =\left(\gamma_{\partial K}^{\mathrm{g}} \circ \Pi_{K}^{k}-\Pi_{\partial K}^{k} \circ \gamma_{\partial K}^{\mathrm{g}}\right) \circ\left(I-\mathcal{E}_{K}^{k+1}\right) .
\end{aligned}
$$

In particular, $\mathbf{R}_{K}^{k+1}\left(\hat{\mathcal{I}}_{K}^{k}(p)\right)=p$ and $\mathrm{S}_{\partial K}^{k}\left(\hat{\mathcal{I}}_{K}^{k}(p)\right)=0$ for all $p \in \mathbb{P}_{k+1, d}$. 
Recalling the duality pairing $\langle\cdot, \cdot\rangle_{F}$ defined in (3.10), the generalization of the bilinear form $n_{\sharp}$ in the context of HHO methods is the bilinear form defined on $\left(V_{\mathrm{S}}+P_{k+1}^{\mathrm{b}}\left(\mathcal{T}_{h}\right)\right) \times \hat{V}_{h, 0}^{k}$ that acts as follows:

$$
n_{\sharp}\left(v, \hat{w}_{h}\right):=\sum_{K \in \mathcal{T}_{h}} \sum_{F \in \mathcal{F}_{K}}\left\langle\left(\boldsymbol{\sigma}(v) \cdot \boldsymbol{n}_{K}\right)_{\mid F},\left(w_{K}-w_{\partial K}\right)_{\mid F}\right\rangle_{F} .
$$

LEmma 4.16 (Identities and boundedness for $n_{\sharp}$ ). The following holds true for all $\hat{w}_{h} \in \hat{V}_{h, 0}^{k}$, all $v_{h} \in P_{k+1}^{\mathrm{b}}\left(\mathcal{T}_{h}\right)$ and all $v \in V_{\mathrm{s}}$ :

$$
\begin{aligned}
n_{\sharp}\left(v_{h}, \hat{w}_{h}\right) & =\sum_{K \in \mathcal{T}_{h}} \int_{K} \lambda_{K} \nabla v_{h \mid K} \cdot \nabla\left(\mathrm{R}_{K}^{k+1}\left(\hat{w}_{K}\right)-w_{K}\right) \mathrm{d} x, \\
n_{\sharp}\left(v, \hat{w}_{h}\right) & =\sum_{K \in \mathcal{T}_{h}} \int_{K}\left(\boldsymbol{\sigma}(v) \cdot \nabla w_{K}+(\nabla \cdot \boldsymbol{\sigma}(v)) w_{K}\right) \mathrm{d} x .
\end{aligned}
$$

Moreover, there is $c$ so that the following holds for all $h \in \mathcal{H}$, all $\lambda \in \Lambda(\Pi)$, all $v \in V_{\mathrm{S}}+P_{k+1}^{\mathrm{b}}\left(\mathcal{T}_{h}\right)$, and all $\hat{w}_{h} \in \hat{V}_{h, 0}^{k}$ :

$$
\left|n_{\sharp}\left(v, \hat{w}_{h}\right)\right| \leq c|v|_{n_{\sharp}}\left(\sum_{K \in \mathcal{T}_{h}} \lambda_{K} h_{K}^{-1}\left\|w_{K}-w_{\partial K}\right\|_{L^{2}(\partial K)}^{2}\right)^{\frac{1}{2}},
$$

with the $|\cdot|_{n_{\sharp}}$-seminorm defined in (3.18).

Proof. (i) We first prove (4.37a). Let $v_{h} \in P_{k+1}^{\mathrm{b}}\left(\mathcal{T}_{h}\right)$ and $\hat{w}_{h} \in \hat{V}_{h, 0}^{k}$. Since the restriction of $\boldsymbol{\sigma}\left(v_{h}\right)$ to each mesh cell is smooth and since the trace on $\partial K$ of the face-to-cell lifting operator $L_{F}^{K}$ is nonzero only on $F$, for all $F \in \mathcal{F}_{K}$, we have

$$
\begin{aligned}
& \left\langle\left(\boldsymbol{\sigma}\left(v_{h}\right) \cdot \boldsymbol{n}_{K}\right)_{\mid F},\left(w_{K}-w_{\partial K}\right)_{\mid F}\right\rangle_{F} \\
& \left.=\int_{K} \boldsymbol{\sigma}\left(v_{h}\right)_{\mid K} \cdot \nabla L_{F}^{K}\left(\left(w_{K}-w_{\partial K}\right)_{\mid F}\right)+\left(\nabla \cdot \boldsymbol{\sigma}\left(v_{h}\right)_{\mid K}\right) L_{F}^{K}\left(\left(w_{K}-w_{\partial K}\right)_{\mid F}\right)\right) \mathrm{d} x \\
& =\int_{\partial K} \boldsymbol{\sigma}\left(v_{h}\right)_{\mid K} \cdot \boldsymbol{n}_{K} L_{F}^{K}\left(\left(w_{K}-w_{\partial K}\right)_{\mid F}\right) \mathrm{d} s=\int_{F} \boldsymbol{\sigma}\left(v_{h}\right)_{\mid K} \cdot \boldsymbol{n}_{K}\left(w_{K}-w_{\partial K}\right) \mathrm{d} s
\end{aligned}
$$

where we used the divergence formula in $K$. Therefore, we obtain

$$
\begin{aligned}
\left.n_{\sharp}\left(v_{h}, \hat{w}_{h}\right)\right) & =\sum_{K \in \mathcal{T}_{h}} \int_{\partial K} \boldsymbol{\sigma}\left(v_{h}\right)_{\mid K} \cdot \boldsymbol{n}_{K}\left(w_{K}-w_{\partial K}\right) \mathrm{d} s \\
& =-\sum_{K \in \mathcal{T}_{h}} \lambda_{K} \int_{\partial K} \nabla v_{h \mid K} \cdot \boldsymbol{n}_{K}\left(w_{K}-w_{\partial K}\right) \mathrm{d} s \\
& =\sum_{K \in \mathcal{T}_{h}} \lambda_{K} \int_{K}\left(\nabla v_{h \mid K} \cdot \nabla\left(\mathrm{R}_{K}^{k+1}\left(\hat{w}_{K}\right)-w_{K}\right)\right) \mathrm{d} x,
\end{aligned}
$$

where we used the definition (4.29) of the local reconstruction operator $\mathrm{R}_{K}^{k+1}$ with the test function $v_{h \mid K} \in \mathbb{P}_{k+1, d}$.

(ii) Let us now prove (4.37b). Let $v \in V_{\mathrm{S}}$ and $\hat{w}_{h} \in \hat{V}_{h, 0}^{k}$. We are going to proceed as in the proof of (3.16b). We consider the mollification operators $\mathcal{K}_{\delta}^{\mathrm{d}}: \boldsymbol{L}^{1}(D) \rightarrow \boldsymbol{C}^{\infty}(\bar{D})$ and $\mathcal{K}_{\delta}^{\mathrm{b}}: L^{1}(D) \rightarrow C^{\infty}(\bar{D})$ introduced in $[22, \S 3.2]$. Let us consider the mollified bilinear form

$$
n_{\sharp \delta}\left(v, \hat{w}_{h}\right):=\sum_{K \in \mathcal{T}_{h}} \sum_{F \in \mathcal{F}_{K}}\left\langle\left(\mathcal{K}_{\delta}^{\mathrm{d}}(\boldsymbol{\sigma}(v)) \cdot \boldsymbol{n}_{K}\right)_{\mid F},\left(w_{K}-w_{\partial K}\right)_{\mid F}\right\rangle_{F} .
$$


By using (3.10) and invoking the approximation properties of the mollification operators and the commuting property (3.17), we infer that $\lim _{\delta \rightarrow 0} n_{\sharp \delta}\left(v, \hat{w}_{h}\right)=n_{\sharp}\left(v, \hat{w}_{h}\right)$. Since the restriction of $\mathcal{K}_{\delta}^{\mathrm{d}}(\boldsymbol{\sigma}(v))$ to each mesh cell is smooth and since $\mathcal{K}_{\delta}^{\mathrm{d}}(\boldsymbol{\sigma}(v)) \in$ $C^{0}(\bar{D})$, we infer that

$$
\begin{aligned}
n_{\sharp \delta}\left(v, \hat{w}_{h}\right) & =\sum_{K \in \mathcal{T}_{h}} \int_{\partial K} \mathcal{K}_{\delta}^{\mathrm{d}}(\boldsymbol{\sigma}(v)) \cdot \boldsymbol{n}_{K}\left(w_{K}-w_{\partial K}\right) \mathrm{d} s=\sum_{K \in \mathcal{T}_{h}} \int_{\partial K} \mathcal{K}_{\delta}^{\mathrm{d}}(\boldsymbol{\sigma}(v)) \cdot \boldsymbol{n}_{K} w_{K} \mathrm{~d} s \\
& =\sum_{K \in \mathcal{T}_{h}} \int_{K}\left(\mathcal{K}_{\delta}^{\mathrm{d}}(\boldsymbol{\sigma}(v)) \cdot \nabla w_{K}+\mathcal{K}_{\delta}^{\mathrm{b}}(\nabla \cdot \boldsymbol{\sigma}(v)) w_{K}\right) \mathrm{d} x,
\end{aligned}
$$

where we used the divergence formula and the commuting property (3.17) in the last line. Letting $\delta \rightarrow 0$, we conclude that $n_{\sharp \delta}\left(v, \hat{w}_{h}\right)$ also tends to the right-hand side of (4.37b) as $\delta \rightarrow 0$. Hence, (4.37b) holds true.

(iii) The proof of (4.38) uses the same arguments as the proof of Lemma 3.5.

Remark 4.17 ((4.37b)). The right-hand side of (4.37b) does not depend on the face-based functions $w_{\partial K}$. This identity will replace the argument in $[16,18]$ invoking the continuity of the normal component of $\boldsymbol{\sigma}(u)$ at the mesh interfaces, which makes sense only when the exact solution is smooth enough, say $\boldsymbol{\sigma}(u) \in \boldsymbol{H}^{r}(D)$ with $r>\frac{1}{2}$.

Let $V_{\sharp}:=V_{\mathrm{S}}+P_{k+1}^{\mathrm{b}}\left(\mathcal{T}_{h}\right)$ be equipped with the seminorm $\|v\|_{V_{\sharp}}:=|v|_{\lambda, p, q}$ defined in (4.2). Notice that $\|v\|_{V_{\sharp}}=0$ implies that $v=0$ if $v$ has zero mean-value in each mesh cell $K \in \mathcal{T}_{h}$; this is the case for instance if one takes $v=u-\mathcal{E}_{h}^{k+1}(u)$. We define the consistency error $\delta_{h}: \hat{V}_{h, 0}^{k} \rightarrow\left(\hat{V}_{h, 0}^{k}\right)^{\prime}$ by setting, for all $\hat{w}_{h} \in V_{h, 0}^{k}$,

$$
\left\langle\delta_{h}\left(\hat{v}_{h}\right), \hat{w}_{h}\right\rangle_{\left(\hat{V}_{h, 0}^{k}\right)^{\prime}, \hat{V}_{h, 0}^{k}}:=\hat{\ell}_{h}\left(\hat{w}_{h}\right)-\hat{a}_{h}\left(\hat{v}_{h}, \hat{w}_{h}\right) .
$$

We define global counterparts of the local operators $\mathrm{R}_{K}^{k+1}, \hat{\mathcal{I}}_{K}^{k}$, and $\mathcal{E}_{K}^{k+1}$, namely $\mathrm{R}_{h}^{k+1}: \hat{V}_{h, 0}^{k} \rightarrow P_{k+1}^{\mathrm{b}}\left(\mathcal{T}_{h}\right), \hat{\mathcal{I}}_{h}^{k}: H_{0}^{1}(D) \rightarrow \hat{V}_{h, 0}^{k}$, and $\mathcal{E}_{h}^{k+1}: H^{1}(D) \rightarrow P_{k+1}^{\mathrm{b}}\left(\mathcal{T}_{h}\right)$, by setting $\mathrm{R}_{h}^{k+1}\left(\hat{v}_{h}\right)_{\mid K}:=\mathrm{R}_{K}^{k+1}\left(\hat{v}_{K}\right), \hat{\mathcal{I}}_{h}^{k}(v)_{\mid K}:=\hat{\mathcal{I}}_{K}^{k}\left(v_{\mid K}\right)$, and $\mathcal{E}_{h}^{k+1}(v)_{\mid K}:=\mathcal{E}_{K}^{k+1}\left(v_{\mid K}\right)$, for all $\hat{v}_{h} \in \hat{V}_{h, 0}^{k}$, all $v \in H^{1}(D)$, and all $K \in \mathcal{T}_{h}$.

Lemma 4.18 (Consistency/boundedness). Let $\delta_{h}$ be defined in (4.39) with $\hat{a}_{h}$ and $\hat{\ell}_{h}$ defined in (4.33). Then, there is $\omega_{\sharp}$ such that

$$
\left\|\delta_{h}\left(\hat{\mathcal{I}}_{h}^{k}(u)\right)\right\|_{\left(\hat{V}_{h, 0}^{k}\right)^{\prime}} \leq \omega_{\sharp}\left\|u-\mathcal{E}_{h}^{k+1}(u)\right\|_{V_{\sharp}},
$$

for all $h \in \mathcal{H}$, all $f \in L^{q}(D)$, and all $\lambda \in \Lambda(\Pi)$, where $u$ is the unique solution to (2.2).

Proof. Since $\boldsymbol{\sigma}(u)=-\lambda \nabla u, \nabla \cdot \boldsymbol{\sigma}(u)=f$, and $u \in V_{\mathrm{S}}$, the identity (4.37b) yields $\hat{\ell}_{h}\left(\hat{w}_{h}\right)=\sum_{K \in \mathcal{T}_{h}} \int_{K} f w_{K} \mathrm{~d} x=\sum_{K \in \mathcal{T}_{h}} a_{K}\left(u, w_{K}\right)+n_{\sharp}\left(u, \hat{w}_{h}\right)$, where $a_{K}\left(u, w_{K}\right):=$ $\int_{K}-\boldsymbol{\sigma}(u) \cdot \nabla w_{K} \mathrm{~d} x$. Using the definition of $\hat{a}_{h}$ in (4.33), then the identity $\mathrm{R}_{K}^{k+1} \circ \hat{\mathcal{I}}_{K}^{k}=$ $\mathcal{E}_{K}^{k+1}$ (see (4.35a)), and finally (4.37a) with $v_{h}=\mathcal{E}_{h}^{k+1}(u)$, we obtain

$$
\begin{aligned}
\hat{a}_{h}\left(\hat{\mathcal{I}}_{h}^{k}(u), \hat{w}_{h}\right)= & \sum_{K \in \mathcal{T}_{h}} a_{K}\left(\mathcal{E}_{K}^{k+1}(u), w_{K}\right)+n_{\sharp}\left(\mathcal{E}_{h}^{k+1}(u), \hat{w}_{h}\right) \\
& +\sum_{K \in \mathcal{T}_{h}} \lambda_{K}\left(h_{\partial K}^{-1} S_{\partial K}^{k}\left(\hat{\mathcal{I}}_{K}^{k}(u)\right), S_{\partial K}^{k}\left(\hat{w}_{K}\right)\right)_{L^{2}(\partial K)} .
\end{aligned}
$$


Subtracting these two identities and using the definition of $\mathcal{E}_{K}^{k+1}(u)$, which implies that $a_{K}\left(u-\mathcal{E}_{K}^{k+1}(u), w_{K}\right)=0$, for all $K \in \mathcal{T}_{h}$, leads to $\left\langle\delta_{h}\left(\hat{\mathcal{I}}_{h}^{k}(u)\right), \hat{w}_{h}\right\rangle_{\left(\hat{V}_{h, 0}^{k}\right)^{\prime}, \hat{V}_{h, 0}^{k}}=\mathfrak{T}_{1}+\mathfrak{T}_{2}$ with

$$
\mathfrak{T}_{1}:=n_{\sharp}\left(u-\mathcal{E}_{h}^{k+1}(u), \hat{w}_{h}\right), \quad \mathfrak{T}_{2}:=-\sum_{K \in \mathcal{T}_{h}} \lambda_{K}\left(h_{\partial K}^{-1} S_{\partial K}^{k}\left(\hat{\mathcal{I}}_{K}^{k}(u)\right), \mathrm{S}_{\partial K}^{k}\left(\hat{w}_{K}\right)_{L^{2}(\partial K)} .\right.
$$

We invoke (4.38) to bound $\mathfrak{T}_{1}$ and observe that $\sum_{K \in \mathcal{T}_{h}} \lambda_{K} h_{K}^{-1}\left\|w_{K}-w_{\partial K}\right\|_{L^{2}(\partial K)}^{2} \leq$ $\left\|\hat{w}_{h}\right\|_{\hat{V}_{h, 0}^{k}}^{2}$ owing to (4.28). For the bound on $\mathfrak{T}_{2}$, we proceed as in $[16,18]$.

Theorem 4.19 (Error estimate). For all $f \in L^{q}(D)$ and all $\lambda \in \Lambda(\Pi)$, let $u$ denote the solution to (2.2), and let $\hat{u}_{h} \in \hat{V}_{h, 0}^{k}$ denote the solution to (4.34) with $\hat{a}_{h}$ and $\hat{\ell}_{h}$ defined in (4.33). Then, there is $c$ so that the following quasi-optimal error estimate holds true for all $h \in \mathcal{H}$, all $f \in L^{q}(D)$, and all $\lambda \in \Lambda(\Pi)$ :

$$
\left\|u-\mathrm{R}_{h}^{k+1}\left(\hat{u}_{h}\right)\right\|_{V_{\sharp}} \leq c\left\|u-\mathcal{E}_{h}^{k+1}(u)\right\|_{V_{\sharp}} .
$$

Moreover, letting $t:=\min (r, k+1), \chi_{t}:=1$ if $t \leq 1$ and $\chi_{t}:=0$ if $t>1$, we have

$$
\begin{aligned}
& \left\|u-\mathrm{R}_{h}^{k+1}\left(\hat{u}_{h}\right)\right\|_{V_{\sharp}} \\
& \quad \leq c\left(\sum_{K \in \mathcal{T}_{h}} \lambda_{K} h_{K}^{2 t}|u|_{H^{1+t}(K)}^{2}+\frac{\chi_{t}}{\lambda_{K}} h_{K}^{2 d\left(\frac{d+2}{2 d}-\frac{1}{q}\right)}\|f\|_{L^{q}(K)}^{2}\right)^{\frac{1}{2}} .
\end{aligned}
$$

Proof. (i) We adapt the proof of Lemma 4.1 to exploit the convergence order of the reconstruction operator. Let us set $\hat{\zeta}_{h}^{k}:=\hat{u}_{h}-\hat{\mathcal{I}}_{h}^{k}(u) \in \hat{V}_{h, 0}^{k}$ so that $\hat{\zeta}_{K}^{k}=\hat{u}_{K}-\hat{\mathcal{I}}_{K}^{k}\left(u_{\mid K}\right)$ for all $K \in \mathcal{T}_{h}$. The coercivity property from Lemma 4.14 and the definition of the consistency error imply that

$$
\alpha\left\|\hat{\zeta}_{h}^{k}\right\|_{\hat{V}_{h, 0}^{k}}^{2} \leq \hat{a}_{h}\left(\hat{\zeta}_{h}^{k}, \hat{\zeta}_{h}^{k}\right)=\left\langle\delta_{h}\left(\hat{\mathcal{I}}_{h}^{k}(u)\right), \hat{\zeta}_{h}^{k}\right\rangle_{\left(\hat{V}_{h, 0}^{k}\right)^{\prime}, \hat{V}_{h, 0}^{k}} \leq\left\|\delta_{h}\left(\hat{\mathcal{I}}_{h}^{k}(u)\right)\right\|_{\left(\hat{V}_{h, 0}^{k}\right)^{\prime}}\left\|\hat{\zeta}_{h}^{k}\right\|_{\hat{V}_{h, 0}^{k}},
$$

which implies that $\left\|\hat{\zeta}_{h}^{k}\right\|_{\hat{V}_{h, 0}^{k}} \leq c\left\|u-\mathcal{E}_{h}^{k+1}(u)\right\|_{V_{\sharp}}$ owing to Lemma 4.18. Invoking the inverse inequality (4.3) in $P_{k+1}^{\mathrm{b}}\left(\mathcal{T}_{h}\right)$ and using the definition of the $\|\cdot\|_{\hat{V}_{h, 0}^{k}}$-norm implies that $\left\|\mathrm{R}_{h}^{k+1}\left(\hat{\zeta}_{h}^{k}\right)\right\|_{V_{\sharp}} \leq c\left\|\lambda^{\frac{1}{2}} \nabla_{h} \mathrm{R}_{h}^{k+1}\left(\hat{\zeta}_{h}^{k}\right)\right\|_{L^{2}(D)} \leq c\left\|\hat{\zeta}_{h}^{k}\right\|_{\hat{V}_{h, 0}^{k}}$. Hence, we have

$$
\left\|\mathrm{R}_{h}^{k+1}\left(\hat{\zeta}_{h}^{k}\right)\right\|_{V_{\sharp}} \leq c\left\|u-\mathcal{E}_{h}^{k+1}(u)\right\|_{V_{\sharp}} .
$$

Moreover, since $\mathrm{R}_{K}^{k+1}\left(\hat{\mathcal{I}}_{K}^{k}(u)\right)=\mathcal{E}_{K}^{k+1}(u)$ for all $K \in \mathcal{T}_{h}$, see (4.35a), we have

$$
u-\mathrm{R}_{h}^{k+1}\left(\hat{u}_{h}\right)=u-\mathcal{E}_{h}^{k+1}(u)-\mathrm{R}_{h}^{k+1}\left(\hat{\zeta}_{h}^{k}\right) .
$$

The estimate (4.41) is now a consequence of the triangle inequality.

(ii) We now prove (4.42). Let us set $\eta^{k+1}:=u-\mathcal{E}_{h}^{k+1}(u)$. We need to bound $\left\|\eta^{k+1}\right\|_{V_{\sharp}}=\left|\eta^{k+1}\right|_{\lambda, p, q}$, i.e., we must estimate $\left\|\nabla \eta^{k+1}\right\|_{\boldsymbol{L}^{2}(K)}, h_{K}^{d\left(\frac{1}{2}-\frac{1}{p}\right)}\left\|\nabla \eta^{k+1}\right\|_{\boldsymbol{L}^{p}(K)}$, and $h_{K}^{d\left(\frac{d+2}{2 d}-\frac{1}{q}\right)}\left\|\Delta \eta^{k+1}\right\|_{L^{q}(K)}$ (see (4.2)). Owing to the optimality property of the elliptic projection and the approximation properties of $\Pi_{K}^{k+1}$, we have

$$
\left\|\nabla \eta^{k+1}\right\|_{\boldsymbol{L}^{2}(K)} \leq\left\|\nabla\left(u-\Pi_{K}^{k+1}(u)\right)\right\|_{\boldsymbol{L}^{2}(K)} \leq c h_{K}^{t}|u|_{H^{1+t}(K)},
$$


for $t=\min (r, k+1)$. Let us now consider the other two terms. Let $\ell:=\lceil t\rceil$, so that $t \leq \ell \leq 1+t$. Notice also that $\ell \leq k+1$, and $\ell \geq 1$ since we assumed that $r>0$. Let us set $\eta^{\ell}:=u-\mathcal{E}_{h}^{\ell}(u)$, so that $\left\|\nabla \eta^{\ell}\right\|_{L^{2}(K)} \leq c h_{K}^{t}|u|_{H^{1+t}(K)}$. Invoking the triangle inequality, an inverse inequality, and the triangle inequality again, we infer that

$$
h_{K}^{d\left(\frac{1}{2}-\frac{1}{p}\right)}\left\|\nabla \eta^{k+1}\right\|_{\boldsymbol{L}^{p}(K)} \leq h_{K}^{d\left(\frac{1}{2}-\frac{1}{p}\right)}\left\|\nabla \eta^{\ell}\right\|_{\boldsymbol{L}^{p}(K)}+c\left(\left\|\nabla \eta^{k+1}\right\|_{\boldsymbol{L}^{2}(K)}+\left\|\nabla \eta^{\ell}\right\|_{\boldsymbol{L}^{2}(K)}\right),
$$

and the two terms between the parentheses are bounded by $c h_{K}^{t}|u|_{H^{1+t}(K)}$. Moreover, invoking (4.16), we obtain

$$
\begin{aligned}
h_{K}^{d\left(\frac{1}{2}-\frac{1}{p}\right)}\left\|\nabla \eta^{\ell}\right\|_{L^{p}(K)} & \leq c\left(\left\|\nabla \eta^{\ell}\right\|_{\boldsymbol{L}^{2}(K)}+h_{K}^{t}\left|\nabla \eta^{\ell}\right|_{\boldsymbol{H}^{t}(K)}\right) \\
& =c\left(\left\|\nabla \eta^{\ell}\right\|_{\boldsymbol{L}^{2}(K)}+h_{K}^{t}|u|_{H^{1+t}(K)}\right) \leq c^{\prime} h_{K}^{t}|u|_{H^{1+t}(K)},
\end{aligned}
$$

since $t \leq \ell$. Similarly, we have

$h_{K}^{d\left(\frac{d+2}{2 d}-\frac{1}{q}\right)}\left\|\Delta \eta^{k+1}\right\|_{L^{q}(K)} \leq h_{K}^{d\left(\frac{d+2}{2 d}-\frac{1}{q}\right)}\left\|\Delta \eta^{\ell}\right\|_{L^{q}(K)}+c\left(\left\|\nabla \eta^{k+1}\right\|_{L^{2}(K)}+\left\|\nabla \eta^{\ell}\right\|_{L^{2}(K)}\right)$.

It remains to estimate $h_{K}^{d\left(\frac{d+2}{2 d}-\frac{1}{q}\right)}\left\|\Delta \eta^{\ell}\right\|_{L^{q}(K)}$. We proceed as in the end of the proof of Theorem 4.10. If $t \leq 1$ (so that $\chi_{t}=1$ ), we have $\ell=1$, and we infer that

$$
h_{K}^{d\left(\frac{d+2}{2 d}-\frac{1}{q}\right)}\left\|\Delta \eta^{\ell}\right\|_{L^{q}(K)}=\lambda_{K}^{-1} h_{K}^{d\left(\frac{d+2}{2 d}-\frac{1}{q}\right)}\|f\|_{L^{q}(K)} .
$$

Otherwise, we have $t>1$ (so that $\chi_{t}=0$ ) and $\ell \geq 2$. Since $t>1$ implies that necessarily $q \geq 2$, we then take $q=2$. Then, using the triangle inequality, an inverse inequality, and the triangle inequality again, we obtain

$$
\begin{aligned}
h_{K}\left\|\Delta \eta^{\ell}\right\|_{L^{q}(K)} \leq & h_{K} \| \Delta\left(u-\Pi_{K}^{\ell}(u) \|_{L^{q}(K)}\right. \\
& +c\left(\left\|\nabla\left(u-\Pi_{K}^{\ell}(u)\right)\right\|_{L^{2}(K)}+\left\|\nabla \eta^{\ell}\right\|_{L^{2}(K)}\right),
\end{aligned}
$$

where $\Pi_{K}^{\ell}$ is the $L^{2}$-orthogonal projection onto $\mathbb{P}_{\ell, d}$. We conclude by invoking the approximation properties of $\Pi_{K}^{\ell}$, recalling that $\left\|\nabla \eta^{\ell}\right\|_{L^{2}(K)} \leq c h_{K}^{t}|u|_{H^{1+t}(K)}$.

5. Extensions to Maxwell's equations. The various techniques presented in this paper can be extended to the context of Maxwell's equations, since arguments similar to those exposed in $\S 3$ can be deployed to define the tangential trace of vectors fields on a face of $K$. Without going into the details, we show in this section how that can be done.

5.1. Lifting and tangential trace. Let $p, q$ be real numbers satisfying (3.2), and let $\varrho \in(2, p]$ be such that $q \geq \frac{\varrho d}{\varrho+d}$. Let $K$ be a cell in $\mathcal{T}_{h}$, and let $F \in \mathcal{F}_{K}$ be a face of $K$. Following [25], we introduce the space

$$
\boldsymbol{Y}^{\mathrm{c}}(F):=\left\{\boldsymbol{\phi} \in \boldsymbol{W}^{\frac{1}{\varrho}, \varrho^{\prime}}(F) \mid \boldsymbol{\phi} \cdot \boldsymbol{n}_{F}=0\right\},
$$

which we equip with the norm $\|\phi\|_{\boldsymbol{Y}^{\mathrm{c}}(F)}:=\|\phi\|_{\boldsymbol{L}^{\varrho^{\prime}(F)}}+h_{F}^{\frac{1}{\rho}}|\phi|_{\boldsymbol{W}^{\frac{1}{\varrho}, \varrho^{\prime}}(F)}$. Then the following result can be established by proceeding as in the proof of Lemma 3.1.

Lemma 5.1 (Face-to-cell lifting). Let $p, q$ satisfy (3.2). Let $\varrho \in(2, p]$ be s.t. $q \geq$ $\frac{\varrho d}{\varrho+d}$. For all $K \in \mathcal{T}_{h}$ and all $F \in \mathcal{F}_{K}$, there exists a lifting operator $E_{F}^{K}: \boldsymbol{Y}^{\mathrm{c}}(F) \rightarrow$ $\boldsymbol{W}^{1, \varrho^{\prime}}(K)$ s.t. $E_{F}^{K}(\phi)_{\mid \partial K \backslash F}=\mathbf{0}$ and $E_{F}^{K}(\phi)_{\mid F}=\boldsymbol{\phi}$, for all $\boldsymbol{\phi} \in \boldsymbol{Y}^{\mathrm{c}}(F)$. Moreover, there exists $c$ so that the following holds for all $h \in \mathcal{H}$, all $K \in \mathcal{T}_{h}$, and all $F \in \mathcal{F}_{K}$ :

$$
\left|E_{F}^{K}(\phi)\right|_{\boldsymbol{W}^{1, p^{\prime}}(K)}+h_{K}^{-1+d\left(\frac{1}{q}-\frac{1}{p}\right)}\left\|E_{F}^{K}(\boldsymbol{\phi})\right\|_{\boldsymbol{L}^{q^{\prime}}(K)} \leq c h_{K}^{-\frac{1}{\varrho}+d\left(\frac{1}{\varrho}-\frac{1}{p}\right)}\|\boldsymbol{\phi}\|_{\boldsymbol{Y}^{\mathrm{c}}(F)} .
$$


With this lifting operator in hand, we can define an extension to the notion of the tangential trace on $F$ of a vector field. To this end, we introduce the functional space

$$
\boldsymbol{S}^{\mathrm{c}}(K):=\left\{\boldsymbol{\tau} \in \boldsymbol{L}^{p}(K) \mid \nabla \times \boldsymbol{\tau} \in \boldsymbol{L}^{q}(K)\right\},
$$

where the superscript ${ }^{\mathrm{c}}$ refers to the fact that the tangential trace is related to the curl operator. We equip $\boldsymbol{S}^{\mathrm{c}}(K)$ with the following dimensionally-consistent norm:

$$
\|\boldsymbol{\tau}\|_{\boldsymbol{S}^{\mathrm{c}}(K)}:=\|\boldsymbol{\tau}\|_{\boldsymbol{L}^{p}(K)}+h_{K}^{1+d\left(\frac{1}{p}-\frac{1}{q}\right)}\|\nabla \times \boldsymbol{\tau}\|_{L^{q}(K)} .
$$

We now define the tangential trace of any field $\boldsymbol{\tau}$ in $\boldsymbol{S}^{\mathrm{c}}(K)$ on the face $F$ of $K$ to be the linear form $\left(\boldsymbol{\tau} \times \boldsymbol{n}_{K}\right)_{\mid F} \in \boldsymbol{Y}^{\mathrm{c}}(F)^{\prime}$ such that

$$
\left\langle\left(\boldsymbol{\tau} \times \boldsymbol{n}_{K}\right)_{\mid F}, \phi\right\rangle_{F}:=\int_{K}\left(\boldsymbol{\tau} \cdot \nabla \times E_{F}^{K}(\boldsymbol{\phi})-(\nabla \times \boldsymbol{\tau}) \cdot E_{F}^{K}(\boldsymbol{\phi})\right) \mathrm{d} x,
$$

for all $\phi \in \boldsymbol{Y}^{\mathrm{c}}(F)$, where $\langle\cdot, \cdot\rangle_{F}$ now denotes the duality pairing between $\boldsymbol{Y}^{\mathrm{c}}(F)^{\prime}$ and $\boldsymbol{Y}^{\mathrm{c}}(F)$. Note that the right-hand side of (5.5) is well-defined owing to Hölder's inequality and (5.2).

The discretization now involves the vector-valued broken finite element space

$$
\boldsymbol{P}_{k}^{\mathrm{b}}\left(\mathcal{T}_{h}\right)=\left\{\boldsymbol{v}_{h} \in \boldsymbol{L}^{\infty}(D) \mid \boldsymbol{v}_{h \mid K} \in \boldsymbol{P}_{K}, \forall K \in \mathcal{T}_{h}\right\},
$$

where $\boldsymbol{P}_{K}:=\left(\psi_{K}\right)^{-1}(\widehat{\boldsymbol{P}}) \subset \boldsymbol{W}^{k+1, \infty}(K),(\widehat{K}, \widehat{\boldsymbol{P}}, \widehat{\Sigma})$ is the reference element, and $\psi_{K}$ is an appropriate transformation. For instance, one can take $\psi_{K}(\boldsymbol{v})=\psi_{K}^{\mathrm{g}}(\boldsymbol{v}):=$ $\boldsymbol{v} \circ \boldsymbol{T}_{K}$ for continuous Lagrange elements and for dG approximation; one can also take $\psi_{K}(\boldsymbol{v})=\boldsymbol{\psi}_{K}^{\mathrm{c}}(\boldsymbol{v}):=\mathbb{J}_{K}^{\mathrm{T}}\left(\boldsymbol{v} \circ \boldsymbol{T}_{K}\right)$ for edge elements $\left(\boldsymbol{\psi}_{K}^{\mathrm{c}}\right.$ is the covariant Piola transformation and $\mathbb{J}_{K}$ the Jacobian of the geometric mapping). For any face $F \in \mathcal{F}_{K}$, we denote by $\boldsymbol{P}_{F}$ the trace of $\boldsymbol{P}_{K}$ on $F$. The following result is the counterpart of Lemma 3.2 .

Lemma 5.2 (Bound on tangential component). Let p, $q$ satisfy (3.2). There exists c so that the following estimate holds true for all $\boldsymbol{v} \in \boldsymbol{S}^{\mathrm{c}}(K)$, all $K \in \mathcal{T}_{h}$, all $F \in \mathcal{F}_{K}$, and all $h \in \mathcal{H}$ :

$$
\left\|\left(\boldsymbol{v} \times \boldsymbol{n}_{K}\right)_{\mid F}\right\|_{Y^{\mathrm{c}}(F)^{\prime}} \leq c h_{K}^{-\frac{1}{\varrho}+d\left(\frac{1}{\varrho}-\frac{1}{p}\right)}\|\boldsymbol{v}\|_{\boldsymbol{S}^{\mathrm{c}}(K)} .
$$

Moreover, we have

$$
\left|\left\langle\left(\boldsymbol{v} \times \boldsymbol{n}_{K}\right)_{\mid F}, \boldsymbol{\phi}_{h}\right\rangle\right| \leq c h_{K}^{d\left(\frac{1}{2}-\frac{1}{p}\right)}\|\boldsymbol{v}\|_{\boldsymbol{S}^{\mathrm{c}}(K)} h_{F}^{-\frac{1}{2}}\left\|\boldsymbol{\phi}_{h}\right\|_{L^{2}(F)},
$$

for all $\phi_{h} \in \boldsymbol{P}_{F}$ s.t. $\phi \cdot \boldsymbol{n}_{F}=0$, all $K \in \mathcal{T}_{h}$, all $F \in \mathcal{F}_{K}$, and all $h \in \mathcal{H}$.

Lemma 5.2 is essential for the error analysis of nonconforming approximation techniques of Maxwell's equations. It is a generalization of Bonito et al. [8, Lem. A3] and Buffa and Perugia [9, Lem. 8.2].

5.2. Definition of $n_{\sharp}^{c}$ and key identities. The consistency analysis of Nitsche's boundary penalty method and of the $\mathrm{dG}$ approximation applied to Maxwel's equations can be done by introducing a bilinear form $n_{\sharp}$ as in $\S 3$. We henceforth assume that the space dimension is either $d=2$ or $d=3$.

We define the notion of diffusive flux by introducing $\boldsymbol{\sigma}: \boldsymbol{H}(\operatorname{curl} ; D) \rightarrow \boldsymbol{L}^{2}(D)$ such that $\boldsymbol{\sigma}(\boldsymbol{v}):=\lambda \nabla \times \boldsymbol{v}$, for any $\boldsymbol{v} \in \boldsymbol{H}(\operatorname{curl} ; D)$. Here, the diffusivity $\lambda$ is either 
the reciprocal of the magnetic permeability or the reciprocal of the electrical conductivity, depending whether one works with the electric field or the magnetic field. The diffusivity is assumed to satisfy the hypotheses introduced in Section 2. We further define

$$
\boldsymbol{V}_{\mathrm{S}}:=\left\{\boldsymbol{v} \in \boldsymbol{H}(\operatorname{curl} ; D) \mid \boldsymbol{\sigma}(\boldsymbol{v}) \in \boldsymbol{L}^{p}(D), \nabla \times \boldsymbol{\sigma}(\boldsymbol{v}) \in \boldsymbol{L}^{q}(D)\right\},
$$

and set $\boldsymbol{V}_{\sharp}:=\boldsymbol{V}_{\mathrm{S}}+\boldsymbol{P}_{k}^{\mathrm{b}}\left(\mathcal{T}_{h}\right)$.

We adopt the same notation as in $\S 3$. Recall that for any $K \in \mathcal{T}_{h}$ and any $F \in \mathcal{F}_{K}$, we have defined $\epsilon_{K, F}=\boldsymbol{n}_{F} \cdot \boldsymbol{n}_{K}= \pm 1$. We consider arbitrary weights $\theta_{K, F}$ satisfying (3.13). We introduce the bilinear form $n_{\sharp}^{\mathrm{c}}:\left(\boldsymbol{V}_{\mathrm{S}}+\boldsymbol{P}_{k}^{\mathrm{b}}\left(\mathcal{T}_{h}\right)\right) \times \boldsymbol{P}_{k}^{\mathrm{b}}\left(\mathcal{T}_{h}\right) \rightarrow \mathbb{R}$ defined as follows:

$$
n_{\sharp}^{\mathrm{c}}\left(\boldsymbol{v}, \boldsymbol{w}_{h}\right):=\sum_{F \in \mathcal{F}_{h}} \sum_{K \in \mathcal{T}_{F}} \epsilon_{K, F} \theta_{K, F}\left\langle\left(\boldsymbol{\sigma}(\boldsymbol{v})_{\mid K} \times \boldsymbol{n}_{K}\right)_{\mid F}, \llbracket \Pi_{F}\left(\boldsymbol{w}_{h}\right) \rrbracket\right\rangle_{F},
$$

where $\Pi_{F}$ is the $\ell^{2}$-orthogonal projection onto the hyperplane tangent to $F$, i.e., $\Pi_{F}\left(\boldsymbol{b}_{h}\right):=\boldsymbol{b}_{h}-\left(\boldsymbol{b}_{h} \cdot \boldsymbol{n}_{K}\right) \boldsymbol{n}_{K}=\boldsymbol{n}_{K} \times\left(\boldsymbol{b}_{h} \times \boldsymbol{n}_{K}\right)$. Notice that (5.10) is meaningful since $\Pi_{F}\left(\boldsymbol{b}_{h}\right)_{\mid F}$ is in $\boldsymbol{W}^{\frac{1}{\varrho}, \varrho^{\prime}}(F)$ and $\Pi_{F}\left(\boldsymbol{b}_{h}\right) \cdot \boldsymbol{n}_{F}=0$, i.e., $\Pi_{F}\left(\boldsymbol{b}_{h}\right) \in \boldsymbol{Y}^{\mathrm{c}}(F)$ for any $F \in \mathcal{F}_{h}$. The following result is the counterpart of Lemma 3.3.

Lemma 5.3 (Identities for $n_{\sharp}^{\mathrm{c}}$ ). The following holds true for any choice of weights $\left\{\theta_{K, F}\right\}_{F \in \mathcal{F}_{h}, K \in \mathcal{T}_{F}}$ and for all $\boldsymbol{w}_{h} \in \boldsymbol{P}_{k}^{\mathrm{b}}\left(\mathcal{T}_{h}\right)$, all $\boldsymbol{v}_{h} \in \boldsymbol{P}_{k}^{\mathrm{b}}\left(\mathcal{T}_{h}\right)$, and all $\boldsymbol{v} \in \boldsymbol{V}_{\mathrm{S}}$ :

$$
\begin{aligned}
& n_{\sharp}^{\mathrm{c}}\left(\boldsymbol{v}_{h}, \boldsymbol{w}_{h}\right)=\sum_{F \in \mathcal{F}_{h}} \int_{F}\left(\left\{\boldsymbol{\sigma}\left(\boldsymbol{v}_{h}\right)\right\}_{\theta} \times \boldsymbol{n}_{F}\right) \cdot \llbracket \Pi_{F}\left(\boldsymbol{w}_{h}\right) \rrbracket \mathrm{d} s, \\
& n_{\sharp}^{\mathrm{c}}\left(\boldsymbol{v}, \boldsymbol{w}_{h}\right)=\sum_{K \in \mathcal{T}_{h}} \int_{K}\left(\boldsymbol{\sigma}(v) \cdot \nabla \times \boldsymbol{w}_{h \mid K}-(\nabla \times \boldsymbol{\sigma}(v)) \cdot \boldsymbol{w}_{h \mid K}\right) \mathrm{d} x .
\end{aligned}
$$

Proof. The proof is similar to that of Lemma 3.3. The proof of (5.11a) is quasiidentical to that of (3.16a). For the proof of (5.11b), one invokes the mollifying operators $\mathcal{K}_{\delta}^{\mathrm{c}}: \boldsymbol{L}^{1}(D) \rightarrow \boldsymbol{C}^{\infty}(\bar{D})$ and $\mathcal{K}_{\delta}^{\mathrm{d}}: \boldsymbol{L}^{1}(D) \rightarrow \boldsymbol{C}^{\infty}(\bar{D})$ introduced in [22, §3.2]. These two operators satisfy the following key commuting property:

$$
\nabla \times\left(\mathcal{K}_{\delta}^{\mathrm{c}}(\boldsymbol{\tau})\right)=\mathcal{K}_{\delta}^{\mathrm{d}}(\nabla \times \boldsymbol{\tau})
$$

for all $\boldsymbol{\tau} \in \boldsymbol{L}^{1}(D)$ s.t. $\nabla \times \boldsymbol{\tau} \in \boldsymbol{L}^{1}(D)$. Then, one uses the identities $\llbracket \boldsymbol{v} \times \Pi_{F}(\boldsymbol{w}) \rrbracket=$ $\{\boldsymbol{v}\}_{\theta} \times \llbracket \Pi_{F}(\boldsymbol{w}) \rrbracket+\llbracket \boldsymbol{v} \rrbracket \times\left\{\Pi_{F}(\boldsymbol{w})\right\}_{\bar{\theta}}, \boldsymbol{n}_{K} \times \Pi_{F}\left(\boldsymbol{w}_{h}\right)=\boldsymbol{n}_{K} \times \boldsymbol{w}_{h}$, and $\nabla \cdot\left(\boldsymbol{w}_{h} \times \boldsymbol{\sigma}(\boldsymbol{v})\right)=$ $\boldsymbol{\sigma}(\boldsymbol{v}) \cdot\left(\nabla \times \boldsymbol{w}_{h}\right)-\boldsymbol{w}_{h} \cdot(\nabla \times \boldsymbol{\sigma}(\boldsymbol{v}))$.

We now establish the boundedness of the bilinear form $n_{\sharp}^{\mathrm{c}}$. Since $\boldsymbol{\sigma}(\boldsymbol{v})_{\mid K} \in \boldsymbol{S}^{\mathrm{c}}(K)$ for all $K \in \mathcal{T}_{h}$ and all $\boldsymbol{v} \in \boldsymbol{V}_{\mathrm{S}}+\boldsymbol{P}_{k}^{\mathrm{b}}\left(\mathcal{T}_{h}\right)$, we equip the space $\boldsymbol{V}_{\mathrm{S}}+\boldsymbol{P}_{k}^{\mathrm{b}}\left(\mathcal{T}_{h}\right)$ with the seminorm

$$
\begin{aligned}
|\boldsymbol{v}|_{h_{\sharp}^{\mathrm{c}}}^{2}:=\sum_{K \in \mathcal{T}_{h}} \lambda_{K}^{-1}\left(h_{K}^{2 d\left(\frac{1}{2}-\frac{1}{p}\right)}\left\|\boldsymbol{\sigma}(\boldsymbol{v})_{\mid K}\right\|_{\boldsymbol{L}^{p}(K)}^{2}\right. & \\
& \left.+h_{K}^{2 d\left(\frac{2+d}{2 d}-\frac{1}{q}\right)}\left\|\nabla \times \boldsymbol{\sigma}(\boldsymbol{v})_{\mid K}\right\|_{\boldsymbol{L}^{q}(K)}^{2}\right) .
\end{aligned}
$$

Lemma 5.4 (Boundedness of $n_{\sharp}^{\mathrm{c}}$ ). Let the weights $\left\{\theta_{K, F}\right\}_{K \in \mathcal{T}_{h}}$ and the coefficients $\left\{\lambda_{F}\right\}_{F \in \mathcal{F}}$ be defined in (3.19) and (3.20). There is $c$ so that the following holds for all $h \in \mathcal{H}$, all $\lambda \in \Lambda(\Pi)$, all $\boldsymbol{v} \in \boldsymbol{V}_{\mathrm{S}}+\boldsymbol{P}_{k}^{\mathrm{b}}\left(\mathcal{T}_{h}\right)$, and all $\boldsymbol{w}_{h} \in \boldsymbol{P}_{k}^{\mathrm{b}}\left(\mathcal{T}_{h}\right)$ :

$$
\left|n_{\sharp}^{\mathrm{c}}\left(\boldsymbol{v}, \boldsymbol{w}_{h}\right)\right| \leq c|\boldsymbol{v}|_{n_{\sharp}^{\mathrm{c}}}\left(\sum_{F \in \mathcal{F}_{h}} \lambda_{F} h_{F}^{-1}\left\|\llbracket \llbracket \Pi_{F}\left(\boldsymbol{w}_{h}\right) \rrbracket\right\|_{\boldsymbol{L}^{2}(F)}^{2}\right)^{\frac{1}{2}} .
$$


With the above tools in hand, one can revisit Buffa and Perugia [9] and greatly simplify the analysis of the dG approximation of Maxwell's equations. One can also extend the work in [24] and analyze Nitsche's boundary penalty technique with edge elements; one can also revisit Bonito et al. [7], where Nitsche's boundary penalty technique has been used in conjunction with Lagrange elements. In all the cases, one then obtains robust error estimates.

\section{References.}

[1] C. Amrouche, C. Bernardi, M. Dauge, and V. Girault. Vector potentials in three-dimensional non-smooth domains. Math. Methods Appl. Sci., 21(9):823864, 1998.

[2] D. N. Arnold. An interior penalty finite element method with discontinuous elements. SIAM J. Numer. Anal., 19:742-760, 1982.

[3] S. Badia, R. Codina, T. Gudi, and J. Guzmán. Error analysis of discontinuous Galerkin methods for the Stokes problem under minimal regularity. IMA J. Numer. Anal., 34(2):800-819, 2014.

[4] C. Bernardi and V. Girault. A local regularization operator for triangular and quadrilateral finite elements. SIAM J. Numer. Anal., 35(5):1893-1916, 1998.

[5] C. Bernardi and F. Hecht. Error indicators for the mortar finite element discretization of the Laplace equation. Math. Comp., 71(240):1371-1403, 2002.

[6] C. Bernardi and R. Verfürth. Adaptive finite element methods for elliptic equations with non-smooth coefficients. Numer. Math., 85(4):579-608, 2000.

[7] A. Bonito, J.-L. Guermond, and F. Luddens. Regularity of the maxwell equations in heterogeneous media and lipschitz domains. J. Math. Anal. Appl., 408:498$512,2013$.

[8] A. Bonito, J.-L. Guermond, and F. Luddens. An interior penalty method with $C^{0}$ finite elements for the approximation of the Maxwell equations in heterogeneous media: convergence analysis with minimal regularity. ESAIM Math. Model. Numer. Anal., 50(5):1457-1489, 2016.

[9] A. Buffa and I. Perugia. Discontinuous Galerkin approximation of the Maxwell eigenproblem. SIAM J. Numer. Anal., 44(5):2198-2226, 2006.

[10] E. Burman and P. Zunino. A domain decomposition method for partial differential equations with non-negative form based on interior penalties. SIAM J. Numer. Anal., 44:1612-1638, 2006.

[11] Z. Cai, X. Ye, and S. Zhang. Discontinuous Galerkin finite element methods for interface problems: a priori and a posteriori error estimations. SIAM J. Numer. Anal., 49(5):1761-1787, 2011.

[12] C. Carstensen and M. Schedensack. Medius analysis and comparison results for first-order finite element methods in linear elasticity. IMA J. Numer. Anal., 35 (4):1591-1621, 2015.

[13] B. Cockburn, D. A. Di Pietro, and A. Ern. Bridging the Hybrid High-Order and Hybridizable Discontinuous Galerkin methods. ESAIM: Math. Model Numer. Anal. (M2AN), 50(3):635-650, 2016.

[14] M. Crouzeix and P.-A. Raviart. Conforming and nonconforming finite element methods for solving the stationary Stokes equations. I. Rev. Française Automat. Informat. Recherche Opérationnelle Sér. Rouge, 7(R-3):33-75, 1973.

[15] D. A. Di Pietro and A. Ern. Mathematical Aspects of Discontinuous Galerkin Methods, volume 69 of Mathématiques \&s Applications. Springer-Verlag, Berlin, 2012.

[16] D. A. Di Pietro and A. Ern. A Hybrid High-Order locking-free method for linear 
elasticity on general meshes. Comput. Meth. Appl. Mech. Engrg., 283:1-21, 2015.

[17] D. A. Di Pietro, A. Ern, and J.-L. Guermond. Discontinuous Galerkin methods for anisotropic semi-definite diffusion with advection. SIAM J. Numer. Anal., 46 (2):805-831, 2008.

[18] D. A. Di Pietro, A. Ern, and S. Lemaire. An arbitrary-order and compactstencil discretization of diffusion on general meshes based on local reconstruction operators. Comput. Meth. Appl. Math., 14(4):461-472, 2014.

[19] M. Dryja. On discontinuous Galerkin methods for elliptic problems with discontinuous coefficients. Comput. Methods Appl. Math., 3(1):76-85, 2003.

[20] M. Dryja, J. Galvis, and M. Sarkis. BDDC methods for discontinuous Galerkin discretization of elliptic problems. J. Complexity, 23(4-6):715-739, 2007.

[21] A. Ern and J.-L. Guermond. Discontinuous Galerkin methods for Friedrichs' systems. I. General theory. SIAM J. Numer. Anal., 44(2):753-778, 2006.

[22] A. Ern and J.-L. Guermond. Mollification in strongly Lipschitz domains with application to continuous and discrete de Rham complexes. Comput. Methods Appl. Math., 16(1):51-75, 2016.

[23] A. Ern and J.-L. Guermond. Finite element quasi-interpolation and best approximation. M2AN Math. Model. Numer. Anal., 51(4):1367-1385, 2017.

[24] A. Ern and J.-L. Guermond. Analysis of the edge finite element approximation of the Maxwell equations with low regularity solutions. Comput. Math. Appl., 75 (3):918-932, 2018.

[25] A. Ern and J.-L. Guermond. Abstract nonconforming error estimates and application to boundary penalty methods for diffusion equations and time-harmonic Maxwell's equations. Comput. Methods Appl. Math., 18(3):451-475, 2018.

[26] A. Ern, A. F. Stephansen, and P. Zunino. A discontinuous Galerkin method with weighted averages for advection-diffusion equations with locally small and anisotropic diffusivity. IMA J. Numer. Anal., 29(2):235-256, 2009.

[27] E. Gagliardo. Caratterizzazioni delle tracce sulla frontiera relative ad alcune classi di funzioni in $n$ variabili. Rend. Sem. Mat. Univ. Padova, 27:284-305, 1957.

[28] P. Grisvard. Elliptic problems in nonsmooth domains, volume 24 of Monographs and Studies in Mathematics. Pitman (Advanced Publishing Program), Boston, MA, 1985.

[29] T. Gudi. A new error analysis for discontinuous finite element methods for linear elliptic problems. Math. Comp., 79(272):2169-2189, 2010.

[30] F. Jochmann. An $H^{s}$-regularity result for the gradient of solutions to elliptic equations with mixed boundary conditions. J. Math. Anal. Appl., 238:429-450, 1999.

[31] M. Li and S. Mao. A new a priori error analysis of nonconforming and mixed finite element methods. Appl. Math. Lett., 26(1):32-37, 2013.

[32] J. Nitsche. Über ein Variationsprinzip zur Lösung von Dirichlet-Problemen bei Verwendung von Teilräumen, die keinen Randbedingungen unterworfen sind. Abh. Math. Sem. Univ. Hamburg, 36:9-15, 1971.

[33] J. Schöberl. Commuting quasi-interpolation operators for mixed finite elements. Technical Report ISC-01-10-MATH, Texas A\&M University, 2001. URL www. isc.tamu.edu/publications-reports/tr/0110.pdf.

[34] A. Veeser and P. Zanotti. Quasi-optimal nonconforming methods for symmetric elliptic problems. I-Abstract theory. SIAM J. Numer. Anal., 56(3):1621-1642, 2018.

[35] A. Veeser and P. Zanotti. Quasi-optimal nonconforming methods for symmetric 
elliptic problems. III-Discontinuous Galerkin and other interior penalty methods. SIAM J. Numer. Anal., 56(5):2871-2894, 2018. 